\title{
Ab initio nonperturbative calculation of physical observables in light-front dynamics. Application to the Yukawa model
}

\author{
V. A. Karmanov, ${ }^{1}$ J.-F. Mathiot, ${ }^{2}$ and A. V. Smirnov ${ }^{1}$ \\ ${ }^{1}$ Lebedev Physical Institute, Leninsky Prospekt 53, 119991 Moscow, Russia \\ ${ }^{2}$ Clermont Université, Laboratoire de Physique Corpusculaire, \\ BP10448, F-63000 Clermont-Ferrand, France
}

\begin{abstract}
We present a coherent and operational strategy to calculate, in a nonperturbative way, physical observables in light-front dynamics. This strategy is based on the decomposition of the state vector of any compound system in Fock components, and on the covariant formulation of light-front dynamics, together with the so-called Fock sector dependent renormalization scheme. We apply our approach to the calculation of the electromagnetic form factors of a fermion in the Yukawa model, in the nontrivial three-body Fock space truncation, for rather large values of the coupling constant. We find that, once the renormalization conditions are properly taken into account, the form factors do not depend on the regularization scale, when the latter is much larger than the physical masses. We then extend the Fock space by including antifermion degrees of freedom.
\end{abstract}

PACS numbers: 11.10.Ef, 11.10.Gh, 11.10.St

\section{INTRODUCTION}

We have developed over the last years a general strategy to calculate physical observables of compound systems in a nonperturbative framework [1 6 . This strategy is based on light-front dynamics (LFD), a relativistic Hamiltonian formalism advocated first by Dirac in 1949 [7. In its original formulation, the state vector of any compound system evolves in the light-front time $\tau=t+z$, instead of the usual time $t$. Since the physical vacuum in LFD is identical to the free vacuum (see e.g. Ref. 8 and references therein), it is then natural to decompose the state vector of a compound system in Fock components, since this decomposition does not include any vacuum fluctuations, but contains physical (asymptotic) states only. The problem of finding the state vector can thus be formulated as a $N$-body problem.

For obvious practical reasons, the Fock decomposition should be truncated to a finite number of states (Fock sectors). This truncation should be strictly controled in order to be able to make reliable predictions for physical observables, order by order in the Fock expansion. This is made possible due to two important breakthroughs:

- The formulation of LFD in a covariant way [9, which enables the strict control of any violation of rotational invariance, when the Fock space is truncated. This formulation, called covariant light-front dynamics (CLFD), has proven to be very powerful in the description of relativistic properties of few-body systems [10].
- The development of an appropriate renormalization procedure - the so-called Fock sector dependent renormalization (FSDR) scheme which enables to calculate regularization scale invariant observables order by order in the Fock expansion [4].

In the simplest, two-body, Fock space truncation, our formalism is equivalent to summing the irreducible block - the fermion self-energy calculated in the second order of perturbation theory - to all orders in the chain approximation. This equivalence is caused by the fact that all the chain type contributions are restricted to the two-body Fock sector. Such a result is a direct consequence of our FSDR scheme and the corresponding renormalization conditions.

The first nontrivial calculation corresponds to the three-body Fock space truncation which incorporates, in the Yukawa model for instance, fluctuations of the state vector involving one fermion $(f)$, one fermion and one boson $(f b)$, one fermion and two bosons $(f b b)$ Fock sectors. This calculation includes overlapping type (divergent) diagrams summed to all orders in the coupling constant.

Within the FSDR framework, the first calculation of a physical observable - the anomalous magnetic moment (AMM) of a fermion in the Yukawa model has been done in Ref. [5], using the Pauli-Villars (PV) regularization scheme, as proposed in Ref. 11. The calculation has shown nice convergence of the results as a function of the regularization scale (the PV boson mass in our calculation) for values of the coupling constant $\alpha \equiv g^{2} / 4 \pi$ of order of 0.2 . For stronger cou- 
pling, $\alpha \sim 0.5$, some dependence (though rather weak) of the AMM on the PV boson mass was detected. While this range of the coupling constant values is not particularly small (as compared for instance to the electromagnetic coupling constant), it shows however that the truncation of the Fock expansion was not completely under control.

We detail in the present study an extension of our previous approach [5], in order to control, order by order in the Fock expansion, the regularization scale invariance of physical observables. Our derivation is based on the full account of the renormalization conditions, using the FSDR scheme. We shall see that in the truncated Fock space, the bare coupling constant and counterterms are no more true constants, but become naturally dependent on one of the kinematical variables, as already emphasized in Ref. 12. This dependence is determined unambiguously by the renormalization conditions and allows to restore rotational invariance broken by the truncation, as well as the independence of observables on the masses of the PV particles, when the latter ones are much larger than the physical masses. In Ref. $[5$ the fact that the bare coupling constant and counterterms are, a priori, functions of kinematical variables was not taken into account in full measure. The calculations were done for fixed values of the kinematical variables.

The kinematical dependence of the bare coupling constant and counterterms is intimately linked to the Fock space truncation. Its explicit form is strongly affected by the Fock space "contents". Thus, in the Yukawa model considered in the three-body $(f+f b+$ $f b b)$ approximation, this dependence is quite sizeable. It is substantially reduced, when antifermion degrees of freedom (d.o.f.), namely, the additional three-body Fock sector $f f \bar{f}$, are taken into account. In leading order of perturbation theory, we show that the inclusion of the antifermion d.o.f. results in the independence of the bare coupling constant and counterterms on the kinematical variables.

The plan of the article is as follows. We recall in Sec. [II the general properties of our formalism. We discuss in Sec. III the renormalization conditions. In Sec. [IV] we obtain a system of renormalized equations for the Fock components in the Yukawa model within the $f+f b+f b b$ Fock space truncation and calculate the fermion electromagnetic form factors. In Sec. $\nabla$ we extend the Fock space by the inclusion of the $f f f$ Fock sector and discuss the role of antiparticle d.o.f. We present our conclusions in Sec. VI. The contribution of antifermion d.o.f. to the equations for the Fock components is given in Appendix A

\section{GENERAL FRAMEWORK}

\section{A. Covariant formulation of light-front dynamics}

In the traditional form of LFD, the state vector of a compound system is defined on the light-front plane $t+z=0$ (with $c=1$ ) rather than on the equal-time plane $t=0$. In order to recover explicitly rotational invariance, the state vector is defined, in CLFD, on the light-front plane of general orientation $\omega \cdot x=0$, where $\omega$ is an arbitrary four-vector restricted by the condition $\omega^{2}=0[9,10]$. The traditional form of LFD is recovered by using $\omega=(1,0,0,-1)$.

The state vector $\phi(p)$ of a particle with the mass $M$ should satisfy the Poincaré group equations, and among them

$$
\hat{P}^{2} \phi(p)=M^{2} \phi(p)
$$

The momentum operator $\hat{P}$ is decomposed, on the light front, in terms of its free and interaction parts:

$$
\hat{P}_{\rho}=\hat{P}_{\rho}^{(0)}+\hat{P}_{\rho}^{i n t},
$$

where, in terms of the interaction Hamiltonian $H^{\text {int }}(x)$,

$$
\hat{P}_{\rho}^{i n t}=\omega_{\rho} \int H^{i n t}(x) \delta(\omega \cdot x) d^{4} x .
$$

According to the general properties of LFD, we decompose the state vector of a physical system in Fock sectors. We have schematically

$$
\begin{aligned}
\phi(p) & =\sum_{n=1}^{\infty} \int d \tilde{D}_{n} \phi_{n}\left(k_{1}, \ldots, k_{n} ; p\right) \\
& \times \delta^{(4)}\left(k_{1}+\ldots+k_{n}-p-\omega \tau_{n}\right)|n\rangle,
\end{aligned}
$$

where $|n\rangle$ is the state containing $n$ free particles with the four-momenta $k_{1}, \ldots, k_{n}$ and $\phi_{n}$ 's are relativistic $n$-body wave functions, the so-called Fock components. The phase space volume element is represented by $d \tilde{D}_{n}$. All the four-momenta are on the corresponding mass shells: $k_{i}^{2}=m_{i}^{2}, p^{2}=M^{2},\left(\omega \tau_{n}\right)^{2}=0$. Note the peculiar overall four-momentum conservation law given by the $\delta$-function. It follows from the general transformation properties of the light-front plane $\omega \cdot x=0$ under four-dimensional translations [10. The scalar quantity $\tau_{n}$ is a measure of how far the $n$-body system is off the energy shell (on the energy shell $\tau_{n}=0$ ). It is completely determined by this conservation law and the on-mass-shell conditions for each 
individual particle momentum. We get

$$
2 \omega \cdot p \tau_{n}=\left(s_{n}-M^{2}\right), \text { with } s_{n}=\left(k_{1}+\ldots+k_{n}\right)^{2} .
$$

The state $|n\rangle$ can be written as

$$
|n\rangle \equiv d^{\dagger}\left(k_{1}\right) d^{\dagger}\left(k_{2}\right) \ldots d^{\dagger}\left(k_{n}\right)|0\rangle,
$$

where $d^{\dagger}$ is a generic notation for the fermion and boson creation operators. To completely determine the state vector, we normalize it according to

$$
\phi\left(p^{\prime}\right)^{\dagger} \phi(p)=2 p_{0} \delta^{(3)}\left(\mathbf{p}^{\prime}-\mathbf{p}\right) .
$$

With the decomposition (4), the normalization condition (7) writes

$$
\sum_{n=1}^{\infty} I_{n}=1
$$

where $I_{n}$ is the contribution of the $n$-body Fock sector to the norm. For the particular case of the Yukawa model, an explicit formula for $I_{n}$ can be found in Ref. 4].

We shall concentrate, in the following, on systems composed of a spin- $1 / 2$ fermion coupled to scalar bosons. It is convenient to introduce, instead of the wave functions $\phi_{n}$, the vertex functions $\Gamma_{n}$ (which we will also refer to as Fock components), defined by

$$
\bar{u}\left(k_{1}\right) \Gamma_{n} u(p)=\left(s_{n}-M^{2}\right) \phi_{n} \equiv 2 \omega \cdot p \tau_{n} \phi_{n},
$$

where $k_{1}$ is the four-momentum of the constituent fermion. When the Fock space is truncated to order $N$ [i. e. in the sums over $n$ in Eqs. (4) and (8) the terms with $n \leq N$ only are retained], it is necessary to keep track of the order of truncation in the calculation of the vertex function. For this purpose, we will denote the latter by $\Gamma_{n}^{(N)}$, but omit the superscript $(N)$ when it is not necessary. $\Gamma_{n}^{(N)}$ is represented graphically by the diagram shown in Fig. 1 .

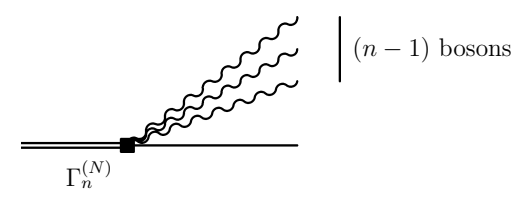

FIG. 1: $n$-body vertex function for the Fock space truncation of order $N$, for a physical fermion (double straight line) made of a constituent fermion (single straight line) coupled to $(n-1)$ bosons (wavy lines).
It is convenient to introduce the notation

$$
\mathcal{G}(p)=2(\omega \cdot p) \hat{\tau} \phi(p),
$$

where $\hat{\tau}$ is the operator which, acting on a given component $\phi_{n}$ of $\phi(p)$, gives $\tau_{n} \phi_{n}$. $\mathcal{G}(p)$ has the Fock decomposition which is obtained from Eq. (4) by the replacement of the wave functions $\phi_{n}$ by the vertex functions $\Gamma_{n}$. We can thus cast the eigenstate equation in the form [1]

$$
\mathcal{G}(p)=\frac{1}{2 \pi} \int\left[-\tilde{H}^{i n t}(\omega \tau)\right] \frac{d \tau}{\tau} \mathcal{G}(p),
$$

where $\tilde{H}^{i n t}$ is the interaction Hamiltonian in momentum space. This equation is quite general and equivalent to the eigenstate equation (1). It is nonperturbative.

The graph technique rules derived in Ref. [10] for the calculation of $S$-matrix elements in CLFD refer precisely to the calculation of matrix elements of $-\tilde{H}^{i n t} /(2 \pi \tau)$. A system of coupled eigenstate equations for the Fock components of the state vector can thus be obtained from Eq. (11) by substituting there [via Eq. [10]] the Fock decomposition (4) and calculating the matrix elements of the operator $-\tilde{H}^{i n t} /(2 \pi \tau)$ in the Fock space.

Since our formalism is explicitly covariant, the spin structure of the wave function $\phi_{n}$ is very simple. Indeed, its construction is of purely kinematical nature [10]. It should incorporate however $\omega$-dependent components. The structure of the two-body components for QED and the Yukawa model was detailed in Ref. 2], while the three-body $(f b b)$ component in the Yukawa model was constructed in Ref. [5]. For the purpose of the present study, we recall here the spin structure of the two-body component in the Yukawa model:

$$
\bar{u}\left(k_{1}\right) \Gamma_{2} u(p)=\bar{u}\left(k_{1}\right)\left[b_{1}+\frac{M \psi}{\omega \cdot p} b_{2}\right] u(p) .
$$

The coefficients $b_{1}$ and $b_{2}$ are scalar functions determined by dynamics.

\section{B. Fock sector dependent renormalization scheme}

In standard renormalization theory, the bare parameters (the whole set of bare coupling constants and counterterms) are determined by relating them to physically observable quantities. To perform this 
strategy in practice, two important questions should be clarified, when using CLFD.

( $i$ ) The explicit form of the relationship between the bare and physical parameters depends on the approximation used. For instance, in perturbation theory the bare parameters are polynomial functions of the physical coupling constant. The term with the maximal degree of the coupling constant is determined within a given order of perturbation, while those of lower degrees are taken unchanged from lower orders. This guarantees that calculated physical observables are regularization scale invariant in any order of the perturbative expansion. In our nonperturbative approach based on the truncated Fock decomposition, an analogous requirement implies that the bare parameters should depend a priori on the Fock sector in which they are considered $[13$.

(ii) In order to express the bare parameters through the physical ones, and vice versa, one should be able to calculate physical observables. In LFD, these ones can not depend on the choice of the orientation of the light-front plane. Such a situation indeed takes place, for instance, order by order in perturbation theory, provided the regularization of divergences in LFD amplitudes is done in a rotationally invariant way 3. In nonperturbative LFD calculations, which always imply Fock space truncation, the dependence on the light-front plane orientation may survive even in calculated physical amplitudes. The identification of such amplitudes with observable quantities becomes therefore ambiguous.

The use of our FSDR scheme in CLFD allows us to answer both questions. In this scheme, each of the original bare parameters has an additional index depending on the number of particles in the Fock sector in which this bare parameter appears. In the Yukawa model the fermion mass counterterm, $\delta m$, and the bare coupling constant $g_{0}$, are thus extended each to a whole sequence:

$$
\begin{aligned}
g_{0} & \rightarrow g_{0 l}, \\
\delta m & \rightarrow \delta m_{l},
\end{aligned}
$$

with $l=1,2, \ldots N$. By definition, $g_{01}=0$ and $\delta m_{1}=0$. For $l \geq 2$, the quantities $g_{0 l}$ and $\delta m_{l}$ are calculated by solving the systems of equations for the vertex functions in the $N=2, N=3, \ldots$ approximations successively.

Besides that, as we shall see in the next section, new counterterms which depend explicitly on the orientation of the light-front plane (i. e. on $\omega$ ) should be introduced in order to restore, if necessary, the ro- tational invariance broken by the truncation. In this case, such counterterms are also mandatory in order to fulfil the renormalization condition. Similarly to the "traditional" counterterms, they have Fock sector dependence. The full set of rules for the calculation of the bare parameters can be found in Refs. [4, 6].

We emphasize that the FSDR scheme is a general method to make nonperturbative calculations in truncated Fock space. It can be easily applied to any physical system admitting particle counting. The Yukawa model studied in the present work has been chosen as an illustration of the capabilities of our approach.

\section{RENORMALIZATION CONDITIONS}

Once the bare coupling constant and the mass counterterms have been identified, one should fix them from a set of renormalization conditions. In perturbation theory, there are three types of quantities to determine: the mass counterterms and the bare coupling constant just mentioned, and the field strength renormalization constants. In the on-mass-shell renormalization scheme, the following conditions are used. The mass counterterms are fixed from the requirement that the two-point Green's functions have a pole at the physical masses. The field normalization constants are fixed from the condition that the residues of the twopoint Green's functions at these poles equal 1 . The bare coupling constant is determined by requiring that the on-mass-shell three-point Green's function is given by the product of the physical coupling constant and the elementary vertex.

The renormalization conditions in LFD are of slightly different form, although they rely on the same grounds. The mass counterterm for each physical state is fixed from the eigenstate equation (1) by demanding that the mass, $M$, of the physical bound state be identical to the constituent mass $m$. The bare coupling constant is determined by relating the onenergy-shell two-body vertex function $\Gamma_{2}$ to the physical coupling constant $g$. Finally, the normalization of the state vector is fixed from the condition (7).

In order to set up the relationship between $\Gamma_{2}$ and the physical coupling constant, one needs to discuss carefully the renormalization factors of the external legs of the two-body vertex function [5, 14. These renormalization factors do also depend on the order of truncation of the Fock space. In the Yukawa model, this relationship reads

$$
\Gamma_{2}^{(N)}\left(s_{2}=M^{2}\right)=g \sqrt{I_{1}^{(N-1)}} \sqrt{Z_{b}} .
$$


Eq. 14 can be interpreted in simple physical terms. Each leg of the two-body vertex function contributes for a different factor $\sqrt{Z}$ to the physical coupling constant, where $Z$ is the field strength normalization factor. The initial fermion state is the physical state normalized to 1 , so that $Z=1$ in that case. The final boson line should be renormalized by a factor $\sqrt{Z_{b}}$. In the approximation where fermionantifermion loop contributions are not considered (the so-called quenched approximation), we have $Z_{b}=1$. Finally, the field strength normalization factor of the constituent fermion is just the weight of the one-body component in the norm of the physical state [5], i.e. $Z=I_{1}$ in that case, according to Eq. (8). Following our FSDR scheme, the normalization factor of the final constituent fermion should correspond to the truncation of order $N-1$ of the Fock space, since there is, by construction of the two-body vertex function, one extra boson in flight in the final state.

Under the PV regularization, PV particles are considered, in the interaction Hamiltonian, on equal grounds with the physical ones. From here it follows that each constituent particle line in the two-body vertex may correspond to either a physical or a PV particle. Observable amplitudes are described by diagrams with physical external legs only. For this reason, the renormalization condition (14) should be imposed on the two-body vertex function with constituent lines corresponding to the physical fermion and boson.

The condition (14) has an important consequence: the two-body vertex function at $s_{2}=M^{2}$ should be independent of the orientation $\omega$ of the light-front plane. With the spin decomposition 12 , this implies that the component $b_{2}$ at $s_{2}=M^{2}$ should be identically zero:

$$
b_{2}^{(N)}\left(s_{2}=M^{2}\right) \equiv 0 .
$$

If Eq. 15) is satisfied, Eq. (14), in the quenched approximation, turns into

$$
b_{1}^{(N)}\left(s_{2}=M^{2}\right) \equiv g \sqrt{I_{1}^{(N-1)}} .
$$

While the property $(15)$ is automatically verified in the case of the two-body Fock space truncation, provided one uses a rotationally invariant regularization scheme 3, 4, this is not guaranteed for higher order truncations. Indeed, nothing prevents $\Gamma_{2}$ to be $\omega$ dependent, since it is an off-shell amplitude, but this dependence must completely disappear on the energy shell. It would be so if no Fock space truncation has been done. The latter results in some $\omega$-dependence of
$\Gamma_{2}$ even on the energy shell. This immediately makes the general renormalization condition (14) ambiguous, since its right-hand side is $\omega$-independent.

Another consequence of the truncation of the Fock space is the fact that the components $b_{1,2}\left(s_{2}=M^{2}\right)$ are not constants. Indeed, $b_{1,2}$ depend a priori on two kinematical variables. For practical purposes, we can take the usual longitudinal momentum fraction $x$ and the transverse (with respect to the three-vector $\boldsymbol{\omega})$ momentum $\mathbf{R}_{\perp}$. They are defined by

$$
\begin{aligned}
x & =\frac{\omega \cdot k_{2}}{\omega \cdot p}, \\
\mathbf{R}_{\perp} & =\mathbf{k}_{2 \perp}-x \mathbf{p}_{\perp},
\end{aligned}
$$

where $k_{2}$ refers to the momentum of the boson in the two-body Fock sector. Note that $\mathbf{R}_{\perp}^{2}=-\left(k_{2}-x p\right)^{2}$ is a relativistic invariant. We have therefore $b_{1,2}=$ $b_{1,2}\left(R_{\perp}, x\right)$.

We denote by $m(\mu)$ the constituent fermion (boson) mass. The on-shell condition

$$
s_{2} \equiv \frac{R_{\perp}^{2}+m^{2}}{1-x}+\frac{R_{\perp}^{2}+\mu^{2}}{x}=M^{2}
$$

can be used to fix one of the two variables, say $R_{\perp}$, in the non-physical domain (for $M=m$ ):

$$
R_{\perp}=R_{\perp}^{*}(x) \equiv i \sqrt{x^{2} m^{2}+(1-x) \mu^{2}},
$$

so that $b_{1,2}^{(N)}\left(s_{2}=M^{2}\right) \equiv b_{1,2}^{(N)}\left(R_{\perp}^{*}(x), x\right)$ calculated in the truncated Fock space depend on $x$ [an example of a particular form of the function $b_{2}^{(N)}\left(R_{\perp}^{*}(x), x\right)$ for $N=3$ is given in Sec. V. Eq. [57]], whereas the conditions (15) and (16) should be valid identically, i.e. for any value of $x$.

In order to enforce the condition (15), we should introduce an appropriate counterterm which depends explicitly on the four-vector $\omega$ 5. It originates from the following additional structure in the interaction Hamiltonian:

$$
\delta \mathcal{H}_{\omega}^{i n t}=-Z_{\omega} \bar{\psi}^{\prime} \frac{m \psi}{i \omega \cdot \partial} \psi^{\prime} \varphi^{\prime},
$$

where $Z_{\omega}$ is just the new counterterm, $\psi^{\prime}\left(\varphi^{\prime}\right)$ is the fermion (scalar boson) field, being a sum of the corresponding physical and PV components, and $1 /(i \omega \cdot \partial)$ is the reversal derivative operator. In the Yukawa model within the three-body approximation the contribution 20 is enough to make all renormalization conditions self-consistent. 
In the truncated Fock space, according to the FSDR rules, $Z_{\omega}$ splits into a sequence of Fock sector dependent contributions $Z_{\omega}^{(l)}$, analogously to the other bare parameters [see Eqs. [13] ]. For the truncation of order $N$, it is supposed that all $Z_{\omega}^{(l)}$ 's with $l=1,2, \ldots N-1$ have been already known from lower order truncations, so that we have to determine the "senior" counterterm $Z_{\omega}^{(N)}$ only. The enforcement of the condition (15), for any $x$, by an appropriate choice of the counterterm $Z_{\omega}^{(N)}$ implies that the latter should a priori depend on $x$, i.e. $Z_{\omega}^{(N)}=Z_{\omega}^{(N)}(x)$. If no Fock space truncation occured, we would get the exact equality $Z_{\omega}=$ const $\equiv 0$, like e.g. in perturbation theory. The same happens for lowest order Fock space truncations because of their triviality. Thus, $Z_{\omega}^{(1)}=0$ by definition. Then, in the two-body approximation, $Z_{\omega}^{(2)}$ is also zero, provided the $\mathrm{PV}$ regularization is used [4]. Nonzero and $x$-dependent counterterms $Z_{\omega}^{(N)}(x)$ appear, starting from $N=3$.

Following the above discussion, the enforcement of the condition (16) induces also a unique dependence of $g_{0 N}=g_{0 N}(x)$ as a function of the kinematical variable $x$.

The fact that, in order to satisfy the renormalization conditions, the bare parameters must depend on the kinematical variable $x$, is crucial to obtain results which are finite after the renormalization procedure in the truncated Fock space is applied. In Sec. IV C, the stability of our results relative to the value of the regularization scale, if the latter reasonably exceeds the physical masses, will be confirmed numerically with high precision.

At first glance, the $x$-dependence of the bare parameters seems, at least, unusual. However, it is a natural consequence of the truncation. Of course, the bare parameters in the fundamental non-truncated Hamiltonian are true constants. After truncation, the initial Hamiltonian is replaced by a finite matrix which acts now in a finite Fock space. But it turns out that the modification of the Hamiltonian is not restricted to a simple truncation. Indeed, to preserve the renormalization conditions, the bare parameters in this finite matrix become $x$-dependent. This $x$-dependence cannot be derived from the initial Lagrangian. It appears only after the Fock space truncation.

Our truncated Hamiltonian with $x$-dependent bare parameters is a self-consistent approximation to the initial fundamental Hamiltonian. One expects that the approximation becomes better, when the number of Fock components increases. At the same time, the $x$-dependence of the bare parameters should become weaker. We will see an indication of that behavior in $\mathrm{Sec}$ VB. We emphasize that there is no any ambiguity in finding the bare parameters, in spite of their $x$-dependence. They are completely fixed from the renormalization conditions.

\section{YUKAWA MODEL IN THE $f+f b+f b b$ APPROXIMATION}

We apply our general strategy to calculate some physical observables for the Yukawa model in the truncated Fock space including sectors with one single fermion, one fermion plus one boson, and one fermion plus two bosons. Previously, we considered the same physical system to calculate the fermion AMM [5], but without $x$-dependent bare parameters. The AMM (as well as any calculated observable) depends on the regularization parameters which are the two PV fermion and boson masses $m_{1}$ and $\mu_{1}$, respectively. In case of a proper renormalization scheme it must tend to a fixed finite value, when both PV masses become much greater than the characteristic physical mass scale. In Ref. [5] we first took the limit $m_{1} \rightarrow \infty$ analytically, just on the level of the equations for the Fock components, and then studied the dependence of the AMM on the remaining PV mass $\mu_{1}$ numerically. We found, at relatively small values of the coupling constant $(\alpha \sim 0.2)$, rather good numerical stability of the AMM as a function of $\mu_{1}$, when $\mu_{1} \gg m, \mu$.

At larger coupling constants $(\alpha \sim 0.5)$, we observed weak but sizable growth of the AMM with the increase of $\mu_{1}$. A possible reason of this uncanceled $\mu_{1}$-dependence of the AMM is the fact that we used constant, i. e. $x$-independent, bare parameters. We shall show in this study that taking into account $x$ dependence of the bare parameters in the truncated Fock space allows to remove completely any dependence of observables on the regularization parameters, even for rather large values of the coupling constant. We shall thus calculate not only the AMM, but both electromagnetic form factors as a function of the momentum transfer squared.

\section{A. Equations for the Fock components}

As shown in Ref. [5], the system of equations for the three vertex functions can be reduced to a closed matrix equation which involves the two-body vertex 
function $\Gamma_{2}$ only. This equation is shown schematically in Fig. 2, Each factor at the vertices is taken according to the FSDR scheme prescriptions. The factors calculated in the three-body approximation, namely, $g_{03}$ and $Z_{\omega}^{(3)}$ appear in the amplitudes involving the one-body vertex function $\Gamma_{1}$ only. Each of the other boson emission and absorbtion vertices in Fig. 2 brings the factor $g_{02}$, since there exists one boson in flight at the time moment corresponding to the vertex and $Z_{\omega}^{(2)}=0$. The mass counterterm contribution $\left(\delta m_{2}\right)$ denoted by the cross appears, in the equation for $\Gamma_{2}$, within the two-body sector only. Analogous contributions inside the three-body sector are absent, because they correspond to the factor $\delta m_{1}$ which is zero.

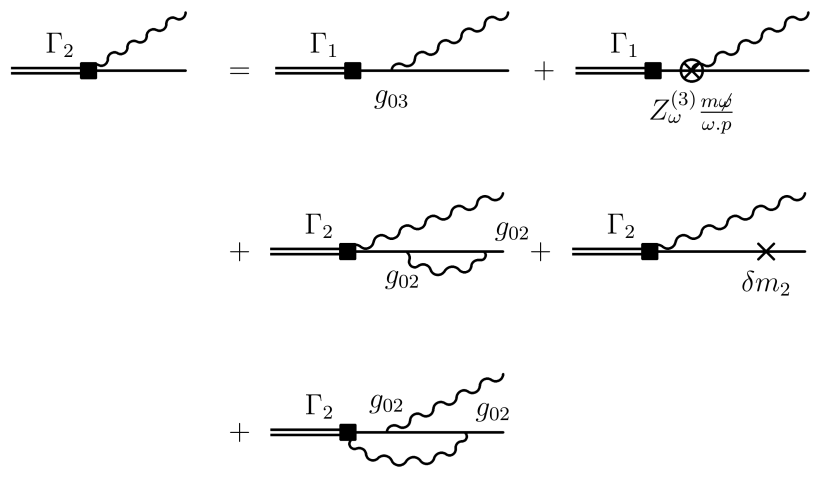

FIG. 2: Equation for the two-body Fock component in the $f+f b+f b b$ approximation.

The fermion-boson loop (see the third diagram on the right-hand side of the graphical equation in Fig. 2p is nothing else than the two-body fermion self-energy. In CLFD, it can be represented as the following general decomposition [3, 5]:

$$
\Sigma(\not k)=g_{02}^{2}\left[\mathcal{A}\left(k^{2}\right)+\mathcal{B}\left(k^{2}\right) \frac{\not k}{m}+\mathcal{C}\left(k^{2}\right) \frac{m \psi}{\omega \cdot k}\right],
$$

where $\mathcal{A}, \mathcal{B}$, and $\mathcal{C}$ are scalar functions given in Ref. [5]. Note that under the PV regularization scheme we have $\mathcal{C}\left(k^{2}\right) \equiv 0$.

Since each of the two constituent lines of the twobody vertex may correspond to either a physical or a PV particle, we have to distinguish four types of $\Gamma_{2}$, depending on its "contents": (i) physical fermion and physical boson; (ii) physical fermion and PV boson; (iii) PV fermion and physical boson; (iv) two PV particles. It is convenient to supply $\Gamma_{2}$ by the two indices, $i$ (for fermion) and $j$ (for boson), reflecting the sort of constituent. Each index may take two values, 0 and 1 , corresponding to a physical or a PV particle, respectively. The substitution $\Gamma_{2} \rightarrow \Gamma_{2}^{i j}$ means the substitutions $b_{1,2} \rightarrow b_{1.2}^{i j}$ for each of its spin components defined by Eq. (12). The particle masses are denoted by $m_{i}$ and $\mu_{j}$ with $m_{0} \equiv m$ and $\mu_{0} \equiv \mu$, by definition.

We shall consider in this study two different orders in taking the infinite limit for the masses of the PV particles. One of them, already considered in Ref. [5, corresponds to the case, when the mass of the PV fermion, $m_{1}$, is first set to infinity (analytically), while the PV boson mass, $\mu_{1}$, is kept finite. In the present section, we re-formulate the system of equations for the Fock components in this limit, taking into account the $x$-dependence of the bare parameters. We then study numerically the dependence of our results on $\mu_{1}$, when the latter is much greater than the physical masses. In addition, we discuss in Sec. IVD the opposite order of limits, when $\mu_{1}$ is first set to infinity.

In order to take the limit $m_{1} \rightarrow \infty$, it is convenient to introduce a set of functions, $h_{i}^{j}$ and $H_{i}^{j}$, which remain finite in this limit. They are given by [5]

$$
b_{1}^{i j}=\frac{m_{i}}{m} h_{i}^{j}, \quad b_{2}^{i j}=\frac{m_{i}}{m} \frac{H_{i}^{j}-\left(1-x+\frac{m_{i}}{m}\right) h_{i}^{j}}{2(1-x)} .
$$

In terms of the functions $h_{i}^{j}$ and $H_{i}^{j}$, the equation shown in Fig. 2 corresponds to the following system 
of integral equations $[5]^{1}$ :

$$
\begin{aligned}
h_{0}^{j}\left(R_{\perp}, x\right)= & g_{03}^{\prime}+g^{\prime 2}\left[K_{1}^{j} h_{0}^{j}\left(R_{\perp}, x\right)+K_{2}^{j} h_{1}^{j}\left(R_{\perp}, x\right)\right] \\
& +g^{\prime 2} i_{0}^{j}\left(R_{\perp}, x\right), \\
h_{1}^{j}\left(R_{\perp}, x\right)= & g^{\prime 2}\left[-K_{3}^{j} h_{0}^{j}\left(R_{\perp}, x\right)+K_{4}^{j} h_{1}^{j}\left(R_{\perp}, x\right)\right] \\
& +g^{\prime 2} i_{1}^{j}\left(R_{\perp}, x\right), \\
H_{0}^{j}\left(R_{\perp}, x\right)= & g_{03}^{\prime}\left[(2-x)+Z_{\omega}^{\prime}(1-x)\right] \\
& +g^{\prime 2}\left[K_{1}^{j} H_{0}^{j}\left(R_{\perp}, x\right)+K_{2}^{j} H_{1}^{j}\left(R_{\perp}, x\right)\right] \\
& +g^{\prime 2} I_{0}^{j}\left(R_{\perp}, x\right), \\
H_{1}^{j}\left(R_{\perp}, x\right)= & g_{03}^{\prime} \\
& +g^{\prime 2}\left[-K_{3}^{j} H_{0}^{j}\left(R_{\perp}, x\right)+K_{4}^{j} H_{1}^{j}\left(R_{\perp}, x\right)\right] \\
& +g^{\prime 2} I_{1}^{j}\left(R_{\perp}, x\right) .
\end{aligned}
$$

In the above equations we use the following notations. The coefficients $K_{1-4}^{j}$ are defined by

$$
\begin{aligned}
K_{1}^{j} & =\frac{1}{m}\left\{\mathcal{B}_{r}\left(s_{1}\right)-\frac{2 m^{2}\left[\mathcal{A}_{r}\left(s_{1}\right)+\mathcal{B}_{r}\left(s_{1}\right)\right]}{m^{2}-s_{1}}\right\}, \\
K_{2}^{j} & =\frac{\mathcal{A}_{r}\left(s_{1}\right)+\mathcal{B}_{r}\left(s_{1}\right)}{m}, \\
K_{3}^{j} & =\frac{m\left[\mathcal{A}_{r}\left(s_{1}\right)+\mathcal{B}_{r}\left(s_{1}\right)\right]}{m^{2}-s_{1}}, \\
K_{4}^{j} & =\frac{\mathcal{B}_{r}\left(s_{1}\right)}{m}
\end{aligned}
$$

where the subtracted fermion self-energy functions are $\mathcal{A}_{r}\left(s_{1}\right)=\mathcal{A}\left(s_{1}\right)-\mathcal{A}\left(m^{2}\right), \mathcal{B}_{r}\left(s_{1}\right)=\mathcal{B}\left(s_{1}\right)-\mathcal{B}\left(m^{2}\right)$, and their argument

$$
s_{1}=-\frac{R_{\perp}^{2}}{x}+(1-x) m^{2}-\frac{1-x}{x} \mu_{j}^{2}
$$

For convenience, we introduce the quantity

$$
g^{\prime 2}=\frac{g^{2}}{1+g^{2} z_{0}}
$$

\footnotetext{
${ }^{1}$ In Ref. [5] we used the functions $\tilde{h}_{i}^{j}$ and $\tilde{H}_{i}^{j}$ which differ from $h_{i}^{j}$ and $H_{i}^{j}$ by their normalization. The present form of the vertex functions is more convenient for the determination of $x$-dependent bare parameters.
}

and re-define the bare parameters by

$$
\begin{aligned}
& g_{03}^{\prime}=g_{03} \alpha_{0}\left(\frac{1-g^{2} \bar{I}_{2}^{(2)}}{1+g^{2} z_{0}}\right), \\
& Z_{\omega}^{\prime}=\frac{2 Z_{\omega}^{(3)}}{g_{03}}-\frac{\alpha_{1}}{\alpha_{0}},
\end{aligned}
$$

where

$$
\bar{I}_{2}^{(2)}=-\frac{\mathcal{B}\left(m^{2}\right)}{m}-z_{0}
$$

with

$$
z_{0}=2 m\left[\mathcal{A}^{\prime}\left(m^{2}\right)+\mathcal{B}^{\prime}\left(m^{2}\right)\right]
$$

The primes at $\mathcal{A}$ and $\mathcal{B}$ denote their derivatives over $s_{1}$. The quantities $\alpha_{0}$ and $\alpha_{1}$ are, respectively, the physical and PV components of the one-body (fermion) vertex, introduced in Ref. [5. We do not need to calculate them explicitly in our study. We can therefore include them into the definitions of $g_{03}^{\prime}$ and $Z_{\omega}^{\prime}$. The quantity $\bar{I}_{2}^{(2)}$, multiplied by $g^{2}$, is nothing else than the norm of the two-body sector, calculated for the two-body $(f+f b)$ Fock space truncation. Transforming the equation for $\Gamma_{2}$ to the system of equations (23), we took into account the values of the bare coupling constant $g_{02}$ and the mass counterterm $\delta m_{2}$, obtained from the previous calculations [4] within the $f+f b$ truncated Fock space ${ }^{2}$ :

$$
\begin{aligned}
g_{02}^{2} & =\frac{g^{2}}{1-g^{2} \bar{I}_{2}^{(2)}}, \\
\delta m_{2} & =g_{02}^{2}\left[\mathcal{A}\left(m^{2}\right)+\mathcal{B}\left(m^{2}\right)\right] .
\end{aligned}
$$

The integral terms describing the contributions of the

\footnotetext{
${ }^{2}$ In Ref. 4 the quantity $\bar{I}_{2}^{(2)}$ was denoted by $J_{2}$. We changed the notation here in order to avoid its confusion with the notation of the electromagnetic current operator (see below).
} 
three-body state to $\Gamma_{2}$ are

$$
\begin{aligned}
i_{n}^{j}\left(R_{\perp}, x\right)= & \int_{0}^{\infty} R_{\perp}^{\prime} d R_{\perp}^{\prime} \int_{0}^{1-x} d x^{\prime} \sum_{i, j^{\prime}=0}^{1}(-1)^{j^{\prime}} \\
& \times\left[c_{n i} h_{i}^{j^{\prime}}\left(R_{\perp}^{\prime}, x^{\prime}\right)+C_{n i} H_{i}^{j^{\prime}}\left(R_{\perp}^{\prime}, x^{\prime}\right)\right], \\
I_{n}^{j}\left(R_{\perp}, x\right)= & \int_{0}^{\infty} R_{\perp}^{\prime} d R_{\perp}^{\prime} \int_{0}^{1-x} d x^{\prime} \sum_{i, j^{\prime}=0}^{1}(-1)^{j^{\prime}} \\
& \times\left[c_{n i}^{\prime} h_{i}^{j^{\prime}}\left(R_{\perp}^{\prime}, x^{\prime}\right)+C_{n i}^{\prime} H_{i}^{j^{\prime}}\left(R_{\perp}^{\prime}, x^{\prime}\right)\right],
\end{aligned}
$$

with $n=0,1$. The coefficients $c, C, c^{\prime}$, and $C^{\prime}$, which depend on $R_{\perp}, R_{\perp}^{\prime}, x, x^{\prime}, j$, and $j^{\prime}$, are given in Ref. [5].

We should now use the renormalization conditions (15) and (16) in order to determine the bare coupling constant $g_{03}^{\prime}$ and the counterterm $Z_{\omega}^{\prime}$. In terms of the functions $h_{i}^{j}$ and $H_{i}^{j}$, these conditions write

$$
\begin{aligned}
h_{0}^{0}\left(R_{\perp}^{*}, x\right) & =g \sqrt{1-g^{2} \bar{I}_{2}^{(2)}}, \\
H_{0}^{0}\left(R_{\perp}^{*}, x\right) & =g \sqrt{1-g^{2} \bar{I}_{2}^{(2)}}(2-x),
\end{aligned}
$$

where $R_{\perp}^{*}$ is defined by Eq. (19). Setting $R_{\perp}=R_{\perp}^{*}$ and $j=0$ in Eqs. 23a) and (23c), we demand the relations (31) to be valid for arbitrary $0<x<1$. As explained in the previous section, this necessitates to substitute $g_{03}^{\prime} \rightarrow g_{03}^{\prime}(x)$ and $Z_{\omega}^{\prime} \rightarrow Z_{\omega}^{\prime}(x)$. Using that at $R_{\perp}=R_{\perp}^{*}$ and $j=0$ we have $s_{1}=m^{2}$, and, hence, $K_{1}^{0}=z_{0}$ and $K_{2}^{0}=0$, we get

$$
\begin{gathered}
g_{03}^{\prime}(x)=g^{\prime 2}\left[\frac{\sqrt{1-g^{2} \bar{I}_{2}^{(2)}}}{g}-i_{0}^{0}\left(R_{\perp}^{*}, x\right)\right], \\
g_{03}^{\prime}(x) Z_{\omega}^{\prime}(x)=g^{\prime 2}\left[\frac{(2-x) i_{0}^{0}\left(R_{\perp}^{*}, x\right)-I_{0}^{0}\left(R_{\perp}^{*}, x\right)}{1-x}\right] .
\end{gathered}
$$

Substituting these quantities back into the system of equations 23), we obtain

$$
\begin{array}{rrl}
h_{0}^{j}\left(R_{\perp}, x\right)=\eta g & +g^{\prime 2}\left[K_{1}^{j} h_{0}^{j}\left(R_{\perp}, x\right)+K_{2}^{j} h_{1}^{j}\left(R_{\perp}, x\right)\right]+g^{\prime 2} \Delta i_{0}^{j}\left(R_{\perp}, x\right), \\
h_{1}^{j}\left(R_{\perp}, x\right)= & g^{\prime 2}\left[-K_{3}^{j} h_{0}^{j}\left(R_{\perp}, x\right)+K_{4}^{j} h_{1}^{j}\left(R_{\perp}, x\right)\right]+g^{\prime 2} i_{1}^{j}\left(R_{\perp}, x\right), \\
H_{0}^{j}\left(R_{\perp}, x\right)=\eta g(2-x)+g^{\prime 2}\left[K_{1}^{j} H_{0}^{j}\left(R_{\perp}, x\right)+K_{2}^{j} H_{1}^{j}\left(R_{\perp}, x\right)\right]+ & +g^{\prime 2} \Delta I_{0}^{j}\left(R_{\perp}, x\right), \\
H_{1}^{j}\left(R_{\perp}, x\right)=\eta g & +g^{\prime 2}\left[-K_{3}^{j} H_{0}^{j}\left(R_{\perp}, x\right)+K_{4}^{j} H_{1}^{j}\left(R_{\perp}, x\right)\right]+g^{\prime 2} \Delta I_{1}^{j}\left(R_{\perp}, x\right),
\end{array}
$$

where

$$
\eta=\frac{\sqrt{1-g^{2} \bar{I}_{2}^{(2)}}}{1+g^{2} z_{0}}
$$

and

$$
\begin{aligned}
& \Delta i_{0}^{j}\left(R_{\perp}, x\right)=i_{0}^{j}\left(R_{\perp}, x\right)-i_{0}^{0}\left(R_{\perp}^{*}, x\right), \\
& \Delta I_{0}^{j}\left(R_{\perp}, x\right)=I_{0}^{j}\left(R_{\perp}, x\right)-I_{0}^{0}\left(R_{\perp}^{*}, x\right), \\
& \Delta I_{1}^{j}\left(R_{\perp}, x\right)=I_{1}^{j}\left(R_{\perp}, x\right)-i_{0}^{0}\left(R_{\perp}^{*}, x\right) .
\end{aligned}
$$

The functions $h_{i}^{j}$ and $H_{i}^{j}$, being a solution of the in- homogeneous system of equations (33), are properly normalized. Once we have carried out the renormalization procedure [resulting in Eqs. [32)], these equations contain only the physical coupling constant $g$, since $g^{\prime}$ is expressed via $g$ by Eq. 25). Let us remind that, in contrast to the results reported in Ref. [5, we do not fix here any particular value of $x$ at which the renormalization conditions are considered. We keep the full $x$-dependence of the bare coupling constant and the $\omega$-dependent counterterm, according to Eqs. (32). 
Each index $i$ and $j$ can take two values, so that we have to deal with eight vertex functions. The convergence of the integrals over $d R_{\perp}^{\prime}$ in the integral terms 30 is ensured by the mutual cancellation of the physical and PV components at $R_{\perp}^{\prime} \rightarrow \infty$ due to the following properties:

$$
\begin{gathered}
h_{i}^{0}\left(R_{\perp} \rightarrow \infty, x\right)=h_{i}^{1}\left(R_{\perp} \rightarrow \infty, x\right), \\
H_{i}^{0}\left(R_{\perp} \rightarrow \infty, x\right)=H_{i}^{1}\left(R_{\perp} \rightarrow \infty, x\right),
\end{gathered}
$$

which automatically follow, when $R_{\perp} \gg \mu_{1}$, from the structure of the equations (33). This is a direct consequence of the PV regularization scheme. However, if one tries to go over to the limit $\mu_{1} \rightarrow \infty$ inside the integrals (i. e. before the integration over $d R_{\perp}^{\prime}$ ), some of them become divergent, unless all the functions $h_{i}^{j}$ and $H_{i}^{j}$ vanish, when $R_{\perp}^{\prime} \rightarrow \infty$. But because of the nonzero free part on the right-hand side of Eqs. (33) there is no any reason to expect such a behavior of the solution. For instance, assuming that $h_{i}^{j}$ and $H_{i}^{j}$ at $R_{\perp}^{\prime}$ of order $\mu_{1}$ or greater have some finite values, we would encounter divergences like $\log \mu_{1}$. The renormalization leading to the subtractions (35) also does not fully prevent from $\mu_{1}$-divergences, because it "improves" the asymptotic $\left(R_{\perp}^{\prime} \rightarrow \infty\right)$ behavior of the integrands only in the two terms, $\Delta i_{0}^{0}\left(R_{\perp}, x\right)$ and $\Delta I_{0}^{0}\left(R_{\perp}, x\right)$ (corresponding to the components with the physical external particles) among the eight ones. As a result, we can not take the limit $\mu_{1} \rightarrow \infty$ directly in the system of equations (33), and the solution essentially depends on $\mu_{1}$.

From a practical point of view, we should solve the equations at large but finite values of $\mu_{1}$, express physical amplitudes (in which, by definition, all the external lines correspond to physical particles) through the vertex functions, and calculate observables. We then repeat these steps, gradually increasing $\mu_{1}$. If the calculated observables, as a function of $\mu_{1}$, tend to stable values within the required accuracy of the numerical calculations, we shall conclude that our renormalization scheme is successful. Let us emphasize that the stability is analyzed with respect to observable quantities only, while the vertex functions, as well as the Fock sector norms may strongly depend on $\mu_{1}$, even if it reasonably exceeds the physical masses. In the following, we will make this procedure numerically, for the calculation of the electromagnetic form factors.

\section{B. Calculation of the electromagnetic form factors}

The general decomposition of the spin- $1 / 2$ electromagnetic vertex (EMV) in CLFD is given by [3 5, 15.

$$
\begin{aligned}
\bar{u}\left(p^{\prime}\right) G^{\rho} u(p)= & e \bar{u}\left(p^{\prime}\right)\left[F_{1} \gamma^{\rho}+\frac{i F_{2}}{2 m} \sigma^{\rho \nu} q_{\nu}\right. \\
& +B_{1}\left(\frac{\phi}{\omega \cdot p} P^{\rho}-2 \gamma^{\rho}\right)+B_{2} \frac{m \omega^{\rho}}{\omega \cdot p} \\
& \left.+B_{3} \frac{m^{2} \phi \omega^{\rho}}{(\omega \cdot p)^{2}}\right] u(p)
\end{aligned}
$$

where $P=p+p^{\prime}, q=p^{\prime}-p, \sigma^{\rho \nu}=i\left(\gamma^{\rho} \gamma^{\nu}-\gamma^{\nu} \gamma^{\rho}\right) / 2$, $e$ is the physical charge, $F_{1}$ and $F_{2}$ are the physical form factors, and $B_{1,2,3}$ are nonphysical contributions. These latter originate from possible breaking of rotational symmetry, caused by the Fock space truncation. Under the condition $\omega \cdot q=0$, all $F_{1,2}, B_{1,2,3}$ depend on $Q^{2} \equiv-q^{2}$ only. The physical form factors can be found according to [15]

$$
\begin{aligned}
e F_{1}= & \frac{\operatorname{Tr}\left[\left(\not p^{\prime}+m\right) \omega_{\rho} G^{\rho}(\not p+m) \phi\right]}{8(\omega \cdot p)^{2}}, \\
e F_{2}= & \frac{m}{2(\omega \cdot p) Q^{2}} \\
& \times \operatorname{Tr}\left[\left(\not p^{\prime}+m\right) \omega_{\rho} G^{\rho}(\not p+m)\left(\frac{m \phi}{\omega \cdot p}-1\right)\right] .
\end{aligned}
$$

Explicit analytical expressions for the two form factors $F_{1}$ and $F_{2}$ in the Yukawa model for the case of the $f+f b+f b b$ Fock space truncation are given in Ref. [5]. For this reason, we will not dwell on technical derivations, but focus on how the FSDR scheme works to renormalize the fermion EMV.

From Eqs. (37) we can see that both physical form factors are determined by the contraction of the EMV with the four-vector $\omega$, sandwiched between the bispinors. It is thus convenient to define the operator

$$
e J(Q)=\frac{\bar{u}\left(p^{\prime}\right) \omega_{\rho} G^{\rho} u(p)}{2(\omega \cdot p)} .
$$

In standard LFD it is nothing else than the pluscomponent of the electromagnetic current. Within the FSDR scheme in truncated Fock space, the operator (38), similarly to the vertex functions, should be supplied with a superscript indicating the order of truncation $N . J^{(N)}(Q)$ can be represented as a superposition of contributions $J_{n}^{(N)}(Q)$ from Fock sectors 
with different numbers $n$ of particles:

$$
e J^{(N)}(Q)=\sum_{n=1}^{N} e_{0(N-n+1)} J_{n}^{(N)}(Q)
$$

where $e_{0(N-n+1)}$ is the electromagnetic bare coupling constant in the $n$-body sector [5]. By definition, $e_{01}=e$. The standard renormalization condition for the EMV

$$
\left.G^{\rho}\right|_{p^{\prime}=p}=e \gamma^{\rho}
$$

written in terms of $J(Q)$ has a very simple form

$$
J(0)=1 .
$$

If the norms $I_{n}$ of all Fock sectors are finite, the following relation must be valid:

$$
J_{n}^{(N)}(0)=I_{n}^{(N)}
$$

for any $N$. The normalization condition (8) just guarantees the property (41). In the Yukawa model however, the norm of each Fock sector is infinite, and the validity of Eq. (42) depends on the regularization scheme. In Ref. 44 it was proved that Eq. (42) held true for any Fock sector containing one fermion and arbitrary number of bosons, provided the PV regularization is used, when $m_{1} \rightarrow \infty$ at finite $\mu_{1}$. Since this is just the case we consider in the present section, we can safely use the relation (42). A direct consequence of the latter is the following result [4]:

$$
e_{0 n}=e,
$$

for any $n$,i. e. the electromagnetic coupling constant is not renormalized at all.

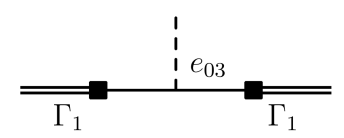

FIG. 3: One-body $(f)$ Fock sector contribution to the electromagnetic vertex.

In the $f+f b+f b b$ approximation, the EMV is given by a sum of contributions shown graphically in Figs. 35. The diagrams in Figs. 3 and 4 correspond, respectively, to the one- and two-body sector contributions to the EMV. The three-body sector contribution in Fig. 5 is expressed, for convenience, through the two-body vertex and separated into two parts (a)

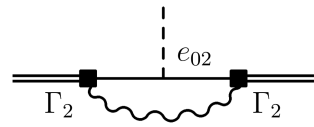

FIG. 4: Two-body $(f b)$ Fock sector contribution to the electromagnetic vertex.

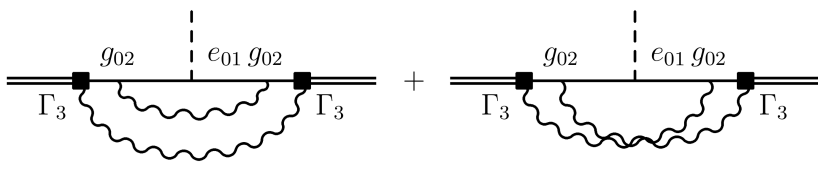

(a)

$(b)$

FIG. 5: Three-body $(f b b)$ Fock sector contributions to the electromagnetic vertex, expressed through the two-body Fock component, with a nested fermion-boson loop (a) and with crossed boson loops (b).

and (b). We will denote these particular contributions to the full operator $J(Q)$ as $J_{1}(Q), J_{2}(Q), J_{3 a}(Q)$, and $J_{3 b}(Q)$. To shorten notations, hereafter we omit the superscript $N$ for all quantities related to the threebody approximation, keeping it however, if a given quantity is calculated within a truncation of another order. From Eq. (39) we find

$$
e J(Q)=e_{03} I_{1}+e_{02} J_{2}(Q)+e\left[J_{3 a}(Q)+J_{3 b}(Q)\right] .
$$

Due to Eqs. 41) and 43, the renormalized $J(Q)$ becomes

$$
\begin{aligned}
J(Q)= & 1+\left[J_{2}(Q)-J_{2}(0)\right] \\
& +\left[J_{3 a}(Q)+J_{3 b}(Q)-J_{3 a}(0)-J_{3 b}(0)\right] .
\end{aligned}
$$

It is convenient to separate the common normalization factor $\sqrt{1-g^{2} \bar{I}_{2}^{(2)}}\left(\bar{I}_{2}^{(2)}\right.$ logarithmically diverges, when $\mu_{1} \rightarrow \infty$ ) from the vertex functions by defining a new set of functions

$$
\left(\begin{array}{c}
\bar{h}_{0,1}^{j} \\
\bar{H}_{0,1}^{j}
\end{array}\right)=\frac{1}{g \sqrt{1-g^{2} \bar{I}_{2}^{(2)}}}\left(\begin{array}{c}
h_{0,1}^{j} \\
H_{0,1}^{j}
\end{array}\right) .
$$

We will supply with a bar each contribution to $J(Q)$, calculated through the functions $\bar{h}_{i}^{j}$ and $\bar{H}_{i}^{j}$ in the vertices. In $J_{3 a}(Q)$ and $J_{3 b}(Q)$ we suppose that the factor $g_{02}^{2}$ coming from the internal vertices in Fig. 5 
is also separated. Eq. 45 transforms thus to

$$
\begin{aligned}
J(Q)= & 1+g^{2}\left(1-g^{2} \bar{I}_{2}^{(2)}\right)\left[\bar{J}_{2}(Q)-\bar{J}_{2}(0)\right] \\
& +g_{02}^{2} g^{2}\left(1-g^{2} \bar{I}_{2}^{(2)}\right) \\
& \times\left[\bar{J}_{3 a}(Q)+\bar{J}_{3 b}(Q)-\bar{J}_{3 a}(0)-\bar{J}_{3 b}(0)\right] .
\end{aligned}
$$

Substituting here $g_{02}^{2}$ from Eq. 29a and rearranging the order of terms, we obtain

$$
\begin{aligned}
J(Q)= & 1+g^{2}\left[\bar{J}_{2}(Q)-\bar{J}_{2}(0)\right] \\
& +g^{4}\left\{\left[\bar{J}_{3 a}(Q)-\bar{I}_{2}^{(2)} \bar{J}_{2}(Q)\right]\right. \\
& \left.-\left[\bar{J}_{3 a}(0)-\bar{I}_{2}^{(2)} \bar{J}_{2}(0)\right]\right\} \\
& +g^{4}\left[\bar{J}_{3 b}(Q)-\bar{J}_{3 b}(0)\right] .
\end{aligned}
$$

Let us emphasize that although Eq. (48) looks like a perturbative expansion, it has no relation to perturbation theory, since the quantities $\bar{J}_{2}, \bar{J}_{3 a}$, and $\bar{J}_{3 b}$ have rather complicated dependence on the coupling constant $g$, governed by the nonperturbative equations for the vertex functions. Nevertheless, it is rather instructive to study how $\mu_{1}$-divergences disappear in Eq. (48), when the vertex functions are given by their perturbative values:

$$
\bar{h}_{0}^{j}=\bar{H}_{1}^{j}=1, \quad \bar{h}_{1}^{j}=0, \quad \bar{H}_{0}^{j}=2-x .
$$

In this case, the $\mu_{1}$-dependence of $\bar{J}_{2}, \bar{J}_{3 a}$, and $\bar{J}_{3 b}$ comes only from the integrals for the EMV. It is easy to show that both $\bar{J}_{2}$ and $\bar{J}_{3 b}$ diverge, when $\mu_{1} \rightarrow \infty$, as $\log \mu_{1}$ with a coefficient independent of $Q$. Subtracting from each of them its value at $Q=0$, we just cancel divergent logarithmic terms, and the result is finite. Concerning the contribution $\bar{J}_{3 a}$ [see Fig. 5 (a)], it has a nested fermion-boson loop which diverges, after the integration over the corresponding kinematical variables, as $\log \mu_{1}$. The integration over the variables of the external loop gives one more $\log \mu_{1}$, so that $\bar{J}_{3 a}$ diverges like $\log ^{2} \mu_{1}$. Its renormalization occurs in two steps, as is seen from the expression in braces in Eq. 48. In a first step, we form the difference $\left[\bar{J}_{3 a}(Q)-\bar{I}_{2}^{(2)} \bar{J}_{2}(Q)\right]$ which kills $\log \mu_{1}$ coming from the nested loop. Indeed, by definition, $\bar{I}_{2}^{(2)}$ coincides, up to a factor of $g^{2}$, with the two-body normalization integral which, according to Eq. 42, is related to the two-body contribution to the EMV by

$$
\bar{J}_{2}^{(2)}(0)=\bar{I}_{2}^{(2)} .
$$

Due to the fact that the divergent part of the nested loop coincides with that in $\bar{J}_{2}^{(2)}(0)$, it is completely canceled in the difference $\left[\bar{J}_{3 a}(Q)-\bar{I}_{2}^{(2)} \bar{J}_{2}(Q)\right]$. In a second step, subtracting from this difference its value at $Q=0$, we remove divergences coming from the integration over the external loop variables. In standard perturbation theory, the renormalization is made by the same scenario. But we emphasize that Eq. (48) naturally appears within the FSDR scheme which is fully nonperturbative.

When the vertex functions are solutions of the system of equations (33) and depend non-trivially on particle momenta and on the coupling constant, it is much more difficult to trace analytically the cancellation of $\mu_{1}$-divergences in calculated observables. For this reason, we will make the corresponding analysis numerically.

\section{Numerical results}

We solve the system of integral equations (33), in order to calculate the two-body Fock component. Knowing the latter, we are able to find the three-body component as well (see Ref. [5] for details). After that, we calculate the electromagnetic form factors by means of Eqs. (37), with the EMV $G^{\rho}$ given by a sum of contributions shown in Figs. 35. The EMV is expressed through the quantity $J(\bar{Q})$ for which we take its renormalized value (48). In all computations, we use the physical particle masses $m=0.938$ and $\mu=0.138$ reflecting the characteristic nuclear physics mass scales. Each physical quantity is calculated for three values of the physical coupling constant, $\alpha=g^{2} / 4 \pi=0.5,0.8$, and 1.0 .

We first compute the fermion AMM which is the value $F_{2}\left(Q^{2}=0\right)$. It is shown in Fig. 6 as a function of the PV boson mass $\mu_{1}$. Each of the two- and threebody Fock sector contributions to the AMM essentially depends on $\mu_{1}$, while their sum is stable, as $\mu_{1}$ becomes large enough. Note that using $x$-dependent bare parameters removes $\mu_{1}$-dependence of the AMM, observed in Ref. [5] already for $\alpha \sim 0.5$, even for larger coupling constants.

In Figs. 7 and 8 , we plot the electromagnetic form factors $F_{1}$ and $F_{2}$, respectively, as a function of $Q^{2}$ for $\mu_{1}=100$, for the three values of the coupling constant, considered here. For this value of $\mu_{1}$, the form factors already reach the zone of stability: further increase of $\mu_{1}$ does not lead to changes distinguishable in the scale of the figures.

We finally show in Fig. 9 the contributions of the one-, two-, and three-body sectors to the norm of the 

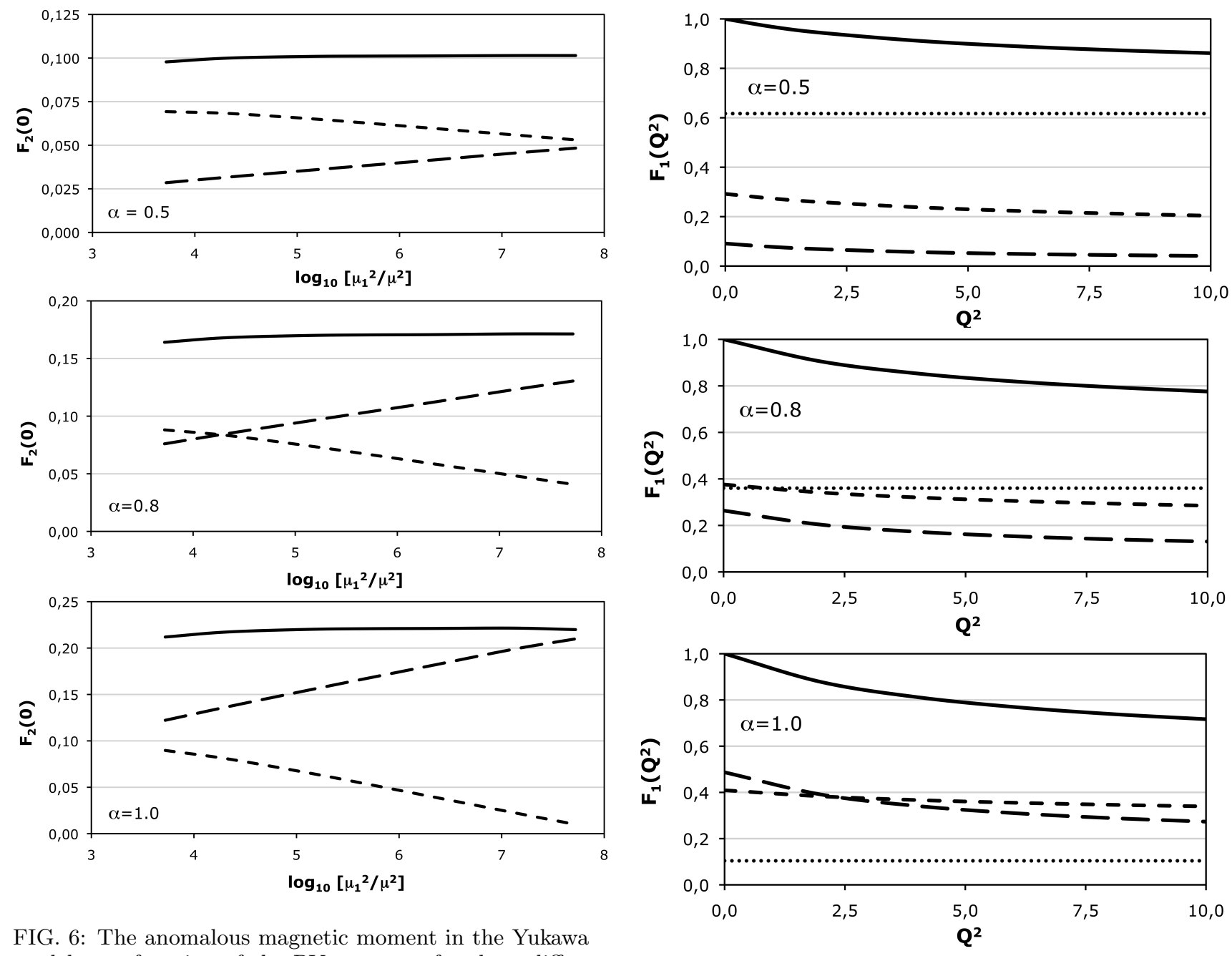

FIG. 6: The anomalous magnetic moment in the Yukawa model as a function of the PV mass $\mu_{1}$, for three different values of the coupling constant, $\alpha=0.5$ (upper plot), 0.8 (middle plot) and $\alpha=1.0$ (lower plot). The dashed and long-dashed lines are, respectively, the two- and threebody contributions, while the solid line is the total result.

state vector. It is interesting to note that for large enough values of the PV boson mass $\mu_{1}$, the norm of the one-body Fock sector gets negative, while the norm of the two-body Fock sector becomes larger than 1 , the sum of all contributions being fixed to 1 by construction. However, physical observables are well defined and do not show any discontinuity, as a function of $\mu_{1}$, when $I_{1}$ changes the sign or $I_{2}$ exceeds unity. This is an illustration of the fact that the norms of the Fock components, in the presence of the PV sectors having negative norms, are not physical observables and, hence, they are expected to be reg-

FIG. 7: Electromagnetic form factor $F_{1}\left(Q^{2}\right)$ in the Yukawa model, at $\mu_{1}=100$, for $\alpha=0.5$ (upper plot), 0.8 (middle plot) and $\alpha=1.0$ (lower plot). The dotted, dashed, and long-dashed lines are, respectively, the one-, two-, and three-body contributions, while the solid line is the total result.

ularization scale dependent. As we can see in these figures, they do depend on $\mu_{1}$, unlike the electromagnetic form factors. The stability of the form factors to variations of the PV masses, when the latter ones are large compared to the physical masses, opens encouraging perspectives for our method of nonperturbative renormalization.

For obvious reasons related to the finite accuracy of numerical calculations, this domain of stability can not be checked up to infinite values of the 

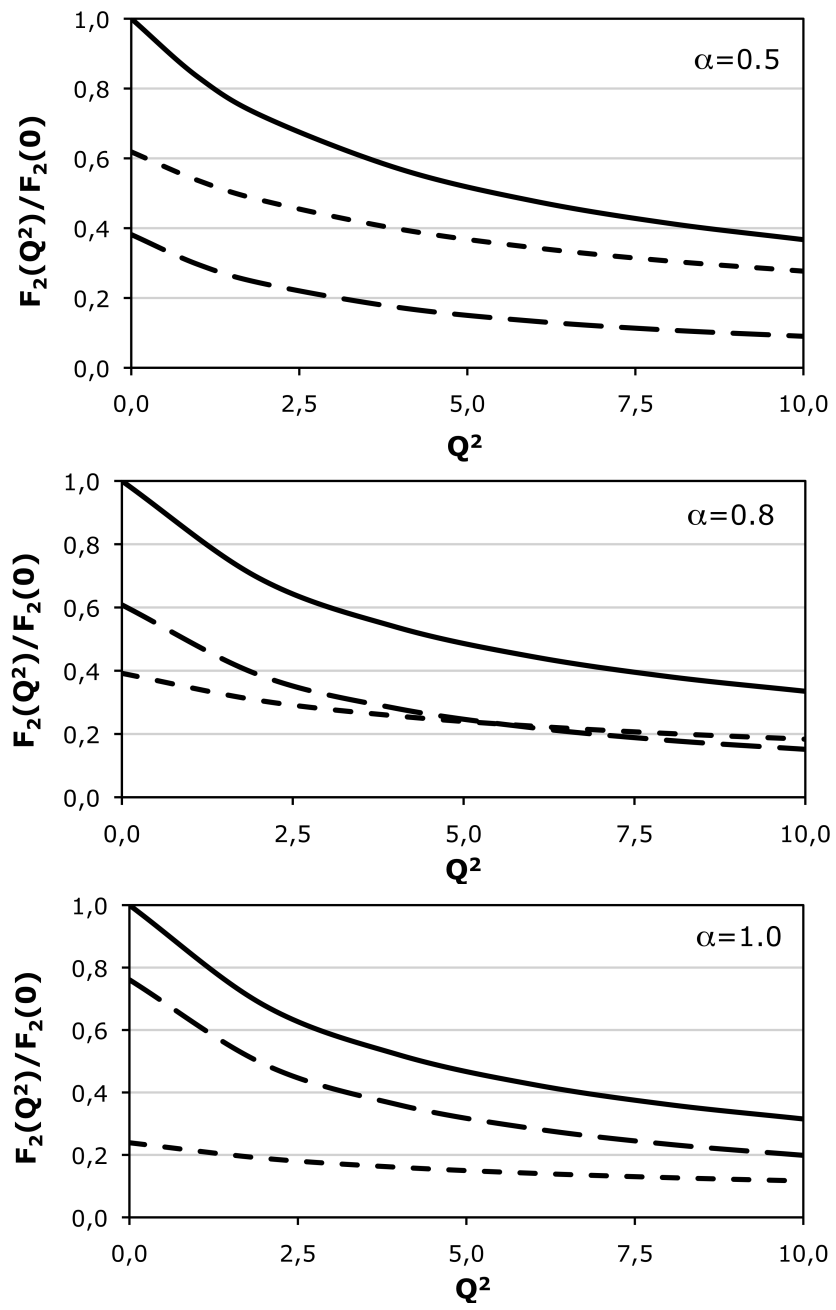

FIG. 8: The same as in Fig. 7, but for the ratio $F_{2}\left(Q^{2}\right) / F_{2}(0)$.

PV mass $\mu_{1}$. However, we have found that for enough large $\mu_{1}$ the stability disappears. Namely, when $\mu_{1}$ approaches some critical mass $\mu_{1 c}$, all the functions $h_{i}^{j}$ and $H_{i}^{j}$ defining the two-body Fock component become very large, when $R_{\perp} \rightarrow \infty$ or $x \rightarrow 0$. At $\mu_{1}=\mu_{1 c}$ these functions are unbounded, because their asymptotic values turn into infinity. Since $h_{i}^{j}, H_{i}^{j}$ start growing from the characteristic values $R_{\perp} \simeq \mu_{1}$ and $x \simeq\left(m / \mu_{1}\right)^{2}$, the calculated observables are sensitive to the variations of $\mu_{1}$, if it is not far enough from $\mu_{1 c}$. The critical mass $\mu_{1 c}$ which can be calculated analytically has very sharp dependence on the coupling constant $\alpha$. For $\alpha \rightarrow 0$ it goes to infinity. As $\alpha$ increases, $\mu_{1 c}$ falls
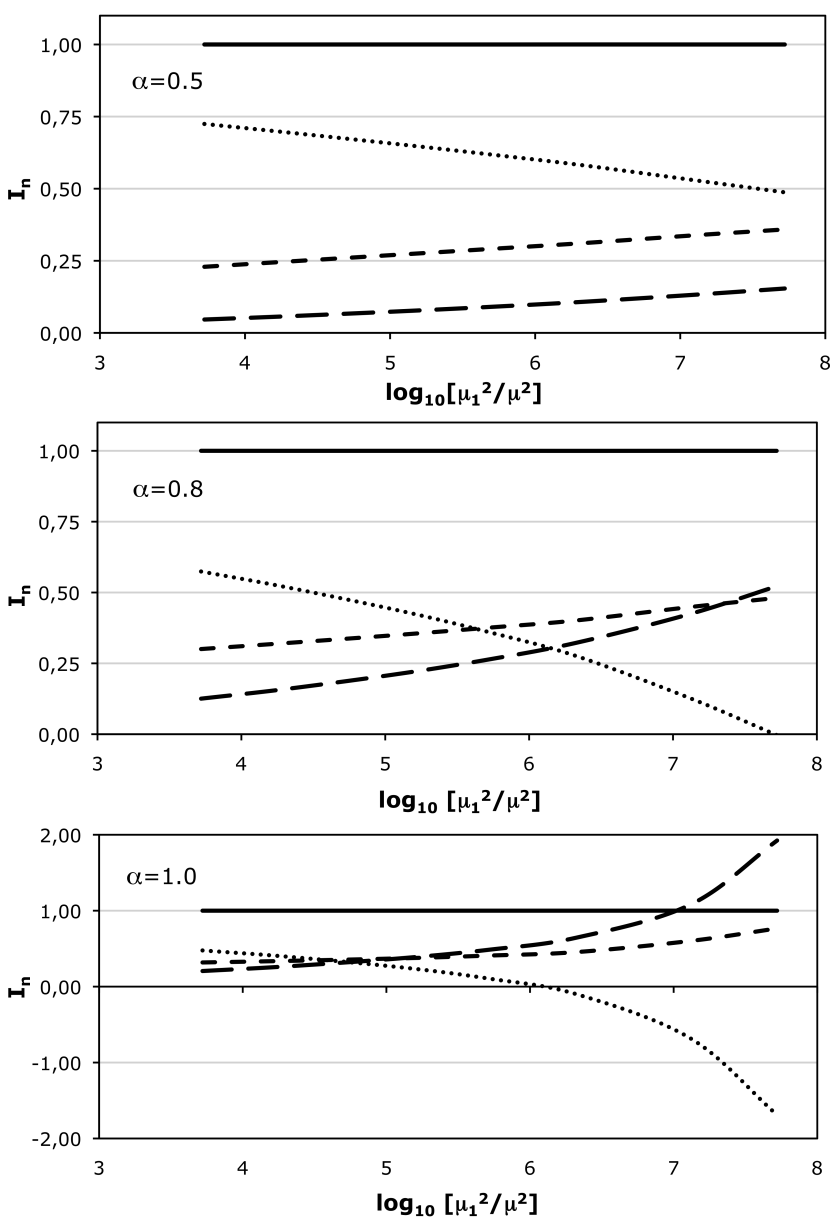

FIG. 9: Individual contributions of the one- (dotted line), two- (dashed line), and three-body (long-dashed line) Fock sectors to the norm (solid line) of the state vector, as a function of the PV boson mass $\mu_{1}$, for $\alpha=0.5$ (upper plot), 0.8 (middle plot), and $\alpha=1.0$ (lower plot).

down very rapidly. Thus, for the physical masses we consider $(m=0.938, \mu=0.138)$ the values of $\mu_{1 c}$, corresponding to $\alpha=0.5,0.8$, and 1 , are, respectively, $5.17 \cdot 10^{8}, 4.17 \cdot 10^{4}$, and $1.8 \cdot 10^{3}$. For little bit larger $\alpha$ 's, $\mu_{1 c}$ becomes comparable with the physical masses. In this domain, a reliable numerical calculation of physical observables is impossible. So, in the Yukawa model, within the three-body truncation and using the PV regularization scheme, the typical dependence of calculated observables on $\mu_{1}$ looks as follows. At $\mu_{1}$ comparable with the physical masses, observables are sensitive to the value of $\mu_{1}$. Then, in the region $m \ll \mu_{1} \ll \mu_{1 c}$ this dependence has a plateau corresponding to the 
zone of stability. This is just our "working" region, inside which we can trust the physical meaning of our numerical results. At $\mu_{1}$ close to $\mu_{1 c}$ we can not expect to perform reliable numerical calculation of physical observables. The determination of the critical mass $\mu_{1 c}$, as well as studying the properties of the system of equations (33), when $\mu_{1}$ is close to $\mu_{1 c}$, go beyond the framework of the present article and will be explained in detail in a future publication.

\section{Additional test of the renormalization scheme}

The system of equations (33) was obtained in the limit $m_{1} \rightarrow \infty$, while $\mu_{1}$ being fixed. We then increase $\mu_{1}$ till the stability of calculated observables is reached. Physical observables however must be independent of the order in which the infinite PV mass limit is taken. For this reason, we shall make a test of the self-consistency of our renormalization procedure. Namely, we perform an additional calculation of the AMM, taking first the limit $\mu_{1} \rightarrow \infty$ (analytically) and then the limit $m_{1} \rightarrow \infty$ (numerically).

The corresponding system of equations for the vertex functions in the limit $\mu_{1} \rightarrow \infty$ can be obtained, analogously to Eqs. (33), from the general equation shown graphically in Fig. 2 Omitting here all technical details, we indicate only the main differences of the system of equations obtained in the limit $\mu_{1} \rightarrow \infty$ from Eqs. 33.

When $\mu_{1} \rightarrow \infty$, the four vertex functions with $j=0$ form a closed sub-system of linear integral equations, while the other four equations involving the functions with $j=1$ can be omitted, since the latter ones do not contribute to the AMM.

As far as the calculation of the electromagnetic form factors is concerned, the change of the order of PV mass limits also brings some new features to the procedure. Eq. (42), and following from it Eq. (43), are not anymore valid. The same relates to Eq. 49 which is a particular case of Eq. 42. As a result, we can not rely on the renormalized expression (48). Indeed, the norm of the two-body sector $I_{2}^{(2)}$ in the two-body approximation was calculated in Ref. 4] [see Eq. (49) there]. Amputating from it the factor $g^{2}$, we find

$$
\begin{aligned}
\bar{I}_{2}^{(2)}= & \frac{1}{8 \pi^{2}} \int_{0}^{\infty} d R_{\perp} R_{\perp} \int_{0}^{1} d x x \sum_{i, j=0}^{1}(-1)^{i+j} \\
& \times \frac{R_{\perp}^{2}+\left[(1-x) m+m_{i}\right]^{2}}{\left[R_{\perp}^{2}+(1-x) \mu_{j}^{2}+x m_{i}^{2}-x(1-x) m^{2}\right]^{2}} .
\end{aligned}
$$

Whereas, for the electromagnetic current $\bar{J}_{2}^{(2)}(0) \equiv$ $J_{2}^{(2)}(0) / g^{2}$, where $J_{2}^{(2)}$ is the two-body component of $J^{(2)}$ defined by Eq. (38) with the EMV $G^{\rho}$ found in the two-body approximation, the calculation gives (the details can be found in Ref. [5]):

$$
\begin{aligned}
\bar{J}_{2}^{(2)}(0)= & \frac{1}{8 \pi^{2}} \int_{0}^{\infty} d R_{\perp} R_{\perp} \int_{0}^{1} d x x \sum_{i, i^{\prime}, j=0}^{1}(-1)^{i+i^{\prime}+j} \\
& \times \frac{R_{\perp}^{2}+(1-x)^{2} m^{2}+(1-x) m\left(m_{i}+m_{i^{\prime}}\right)+m_{i} m_{i^{\prime}}}{\left[R_{\perp}^{2}+(1-x) \mu_{j}^{2}+x m_{i}^{2}-x(1-x) m^{2}\right]\left[R_{\perp}^{2}+(1-x) \mu_{j}^{2}+x m_{i^{\prime}}^{2}-x(1-x) m^{2}\right]} .
\end{aligned}
$$

Both integrals 50 (the two-body norm) and (51) (the two-body current at zero momentum transfer) converge in the limits $m_{1} \rightarrow \infty$ for finite $\mu_{1}$ and $\mu_{1} \rightarrow \infty$ for finite $m_{1}$. As mentioned above, in the limit $m_{1} \rightarrow \infty$ for finite $\mu_{1}$, they coincide with each other and satisfy the relation (49). Taking the opposite limit $\mu_{1} \rightarrow \infty$ for finite $m_{1}$ means retaining only the terms with $j=0$ (the physical boson index) in the sums over $j$. One can easily check that in this limit the difference $\bar{J}_{2}^{(2)}(0)-\bar{I}_{2}^{(2)}$ is not zero. For large values of $m_{1}$, it tends to a finite, mass independent, value:

$$
\bar{J}_{2}^{(2)}(0)-\bar{I}_{2}^{(2)}=\frac{1}{16 \pi^{2}}+O\left(\frac{m}{m_{1}} \log \frac{m_{1}}{m}\right),
$$

while both $\bar{J}_{2}^{(2)}(0)$ and $\bar{I}_{2}^{(2)}$ taken separately diverge as $\log \left(m_{1} / m\right)$, when $m_{1} \rightarrow \infty$. So, under the PV reg- 
ularization scheme, the contribution of a particular Fock sector to the charge form factor at zero momentum transfer may not coincide with the norm of this sector.

To get a renormalized expression for $J(Q)$, we repeat the same steps as in Sec. IVB but without using Eqs. (43) and (49). From the renormalization condition (41) considered, by turn, in the two- and threebody truncated Fock spaces, we determine the bare electromagnetic coupling constants $e_{02}$ and $e_{03}$. Now both of them differ from the physical charge $e$. Substituting them into the general formula (44), we arrive at the result for the renormalized $J(Q)$, which differs from Eq. 48 by the substitution $\bar{I}_{2}^{(2)} \rightarrow \bar{J}_{2}^{(2)}(0)$.

We compare in Table I the numerical results for the AMM, obtained in this way, with those found in Sec. IV C in the limit $m_{1} \rightarrow \infty$. The AMM is considered as a function of the PV mass which is kept finite $\left(m_{1}\right.$, if the limit $\mu_{1} \rightarrow \infty$ has been taken, and vice versa). For convenience of the comparison, we took the same sets of finite PV mass values.

TABLE I: The anomalous magnetic moment calculated for $\alpha=0.8$ in the two different limits of the PV masses.

\begin{tabular}{|c|c|c|}
\hline $\begin{array}{c}\text { PV mass kept } \\
\text { finite }\left(\mu_{1} \text { or } m_{1}\right)\end{array}$ & $\begin{array}{c}\text { AMM when } \\
m_{1} \rightarrow \infty\end{array}$ & $\begin{array}{c}\text { AMM when } \\
\mu_{1} \rightarrow \infty\end{array}$ \\
\hline 5 & 0.1549 & 0.1454 \\
\hline 10 & 0.1641 & 0.1630 \\
\hline 25 & 0.1690 & 0.1704 \\
\hline 50 & 0.1702 & 0.1715 \\
\hline 100 & 0.1706 & 0.1716 \\
\hline 250 & 0.1708 & 0.1714 \\
\hline 500 & 0.1709 & 0.1713 \\
\hline
\end{tabular}

If each of the finite PV masses is much larger than all physical masses, the values of the AMM, obtained in both limits, coincide within the computational accuracy (about $0.2 \%$ ), as it should be if the renormalization procedure works properly. We can thus choose any convenient order of the infinite PV mass limits. Since the equations for the Fock components are technically simpler in the limit $m_{1} \rightarrow \infty$, we continue working with the vertex functions and the EMV taken in this limit. The independence of physical results on the order in which the infinite PV mass limit is taken and, hence, on the way we use to get rid of the bare parameters, is a strong evidence of the self-consistency of our renormalization scheme.

\section{ANTIPARTICLE DEGREES OF FREEDOM}

\section{A. Contribution to the two-body vertex function}

We can extend the Fock decomposition of the fermion state vector by introducing the antifermion d.o.f. In the lowest (also three-body) approximation this corresponds to adding the $f f \bar{f}$ Fock sector to those previously introduced $(f, f b$, and $f b b)$. We have already considered the role of the three-body Fock sector with an antiparticle within the pure scalar model (a heavy scalar boson interacting with light scalar bosons) in Ref. [6]. We perform here a similar study in the Yukawa model.

The antifermion d.o.f. contributions to the twobody vertex are of the following two types:

(i) The first one corresponds to standard fermionantifermion polarization corrections to a boson line, as shown in Fig. 10.

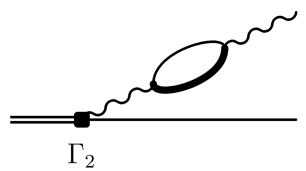

FIG. 10: Contribution of the $f f \bar{f}$ Fock sector to the twobody vertex: polarization correction to the boson line. The antifermion is shown by the thick line.

(ii) The second one corresponds to transition amplitudes $f b \rightarrow f b$ associated to the excitation of antifermion d.o.f. from a fermion line, as shown in Fig. 11(a).

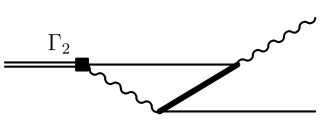

(a)

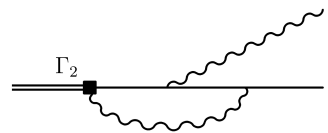

(b)
FIG. 11: Contributions of the $f f \bar{f}$ (a) and $f b b$ (b) Fock sectors to the two-body vertex due to the transition amplitude $f b \rightarrow f b$.

These two contributions $(i)$ and (ii) have different nature. The first one is the $f \bar{f}$ loop. The second contribution is the fermion-boson loop. In addition, the diagram in Fig. 11(a), by changing the order of vertices with respect to the light-front time, evolves to the 
$f b b$ Fock sector contribution, as shown in Fig. 11(b). We shall consider, in this paper, the contributions of the second kind only, which corresponds to the quenched approximation in LFD.

The system of equations for the Fock components can be obtained by direct generalization of the procedure exposed in Ref. [6] for the scalar case. We introduce one more three-body $(f f \bar{f})$ Fock component, in addition to the $f, f b$, and $f b b$ ones considered in Sec. IV] In the three-body approximation, this new Fock component is easily expressed through the twobody component, as well as the $f b b$ one. As a result, we obtain a closed (matrix) equation for the two-body vertex function, as given, in the quenched approximation, by Fig. 12, It differs from the equation in the $f+f b+f b b$ approximation, shown in Fig. 2, by an additional term on the right-hand side (the last diagram in Fig. 12).

We should pay attention to the fact of using the same constant, $g_{02}$, in the elementary vertices $f \leftrightarrow$ $f+b$ and $b \leftrightarrow f+\bar{f}$. Strictly speaking, this is not mandatory from the point of view of the general FSDR rules, because contributions from $f b b$ and $f f \bar{f}$ states represent different Fock sectors. Nevertheless, we assign to these vertices the same factor $g_{02}$, which seems quite natural in this first study of the influence of antifermion d.o.f.

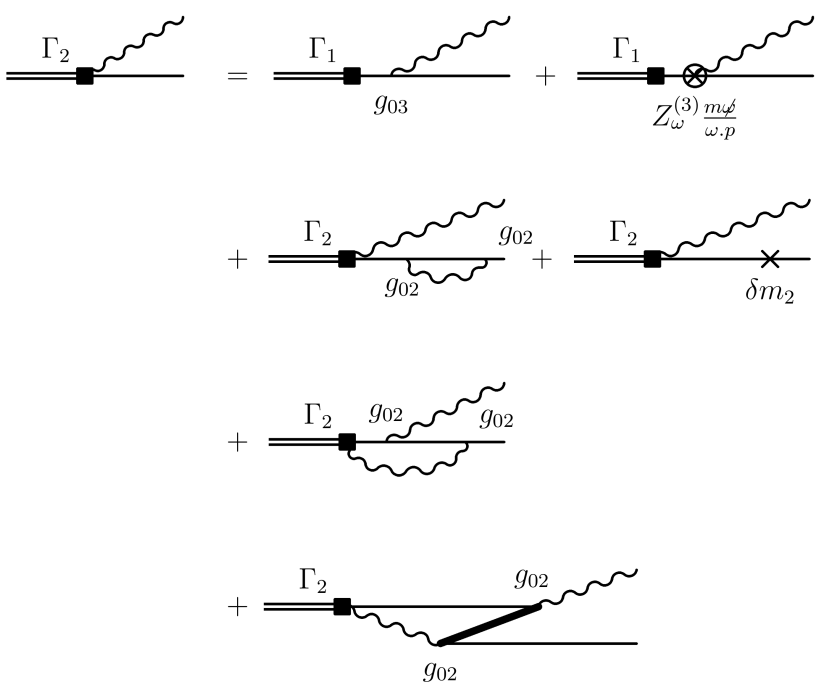

FIG. 12: Graphical representation of the equation for the two-body vertex function $\Gamma_{2}^{(3)}$ including the contribution of antifermion d.o.f. in the quenched approximation.

Since the general structure 12 of the two-body vertex function in the Yukawa model is universal, and since we do not consider in this study polarization corrections to boson lines, the renormalization condition (16) is untouched. The second condition (15) also does not change, because it is universal. We can thus proceed further in the same way as in Sec. IVA. The form of the system of equations for the functions $h_{i}^{j}$ and $H_{i}^{j}$ remains the same as in Eqs. (33), but the integral terms $i_{0}^{j}$ and $I_{0}^{j}$ [see Eqs. (30)] obtain additive $j$-independent contributions according to

$$
i_{0}^{j} \rightarrow i_{0}^{j}+\bar{i}_{0}, \quad I_{0}^{j} \rightarrow I_{0}^{j}+\bar{I}_{0}
$$

where

$$
\begin{aligned}
\bar{i}_{0}\left(R_{\perp}, x\right)= & \int_{0}^{\infty} R_{\perp}^{\prime} d R_{\perp}^{\prime} \int_{1-x}^{1} d x^{\prime} \sum_{j^{\prime}=0}^{1}(-1)^{j^{\prime}} \\
& \times\left[\bar{c}_{0} h_{0}^{j^{\prime}}\left(R_{\perp}^{\prime}, x^{\prime}\right)+\bar{C}_{0} H_{0}^{j^{\prime}}\left(R_{\perp}^{\prime}, x^{\prime}\right)\right], \\
\bar{I}_{0}\left(R_{\perp}, x\right)= & \int_{0}^{\infty} R_{\perp}^{\prime} d R_{\perp}^{\prime} \int_{1-x}^{1} d x^{\prime} \sum_{j^{\prime}=0}^{1}(-1)^{j^{\prime}} \\
& \times\left[\bar{c}_{0}^{\prime} h_{0}^{j^{\prime}}\left(R_{\perp}^{\prime}, x^{\prime}\right)+\bar{C}_{0}^{\prime} H_{0}^{j^{\prime}}\left(R_{\perp}^{\prime}, x^{\prime}\right)\right] .
\end{aligned}
$$

The integral terms $i_{1}^{j}$ and $I_{1}^{j}$ do not change. The coefficients $\bar{c}_{0}, \bar{C}_{0}, \bar{c}_{0}^{\prime}$, and $\bar{C}_{0}^{\prime}$ determining antifermion contributions are given in Appendix A. Note that the limits of the integration over $d x^{\prime}$ in Eqs. (54) differ from those in Eqs. 30.

\section{B. Numerical results}

We have solved numerically the system of equations (33) with the integral terms modified according to Eq. (53), for the same set of parameters as in Sec. IV C $m=0.938, \mu=0.138$, and $\alpha=0.5,0.8$, and 1.0. Along with the functions $h_{i}^{j}$ and $H_{i}^{j}$, we calculate also the bare parameters $g_{03}^{\prime}$ and $Z_{\omega}^{\prime}$ defined by Eqs. (26a) and (26b), respectively. In truncated Fock space, both of them are functions of $x$, according to Eqs. (32), where the integral terms include now antifermion contributions. Besides that, they depend also on the PV mass $\mu_{1}$.

We plot in Figs. 13 and 14 these bare parameters as a function of $x$, each for $\alpha=0.5,0.8$, and 1.0, at a typical value $\mu_{1}=100$. In Fig. 13 the relative value of $g_{03}^{\prime}$ with respect to its mean value $\bar{g}_{03}^{\prime}$ over the interval $0 \leq x \leq 1$ is shown, i.e. we plot the quantity

$$
\delta g_{03}^{\prime}(x)=\left[g_{03}^{\prime}(x)-\bar{g}_{03}^{\prime}\right] / \bar{g}_{03}^{\prime},
$$


where $\bar{g}_{03}^{\prime}=\int_{0}^{1} g_{03}^{\prime}(x) d x$. For comparison, we show also on these plots the same functions calculated without antifermion contributions. The most interesting fact is that the function $g_{03}^{\prime}(x)$, which exhibits strong $x$-dependence in the $f+f b+f b b$ approximation, becomes almost a constant, if the $f f \bar{f}$ Fock sector is included. Concerning the function $Z_{\omega}^{\prime}(x)$, it shows a similar tendency as well, with a bit stronger $x$ dependency than $g_{03}^{\prime}(x)$. In addition, the magnitude of $Z_{\omega}^{\prime}(x)$ is reasonably smaller than that calculated in the $f+f b+f b b$ truncated Fock space.
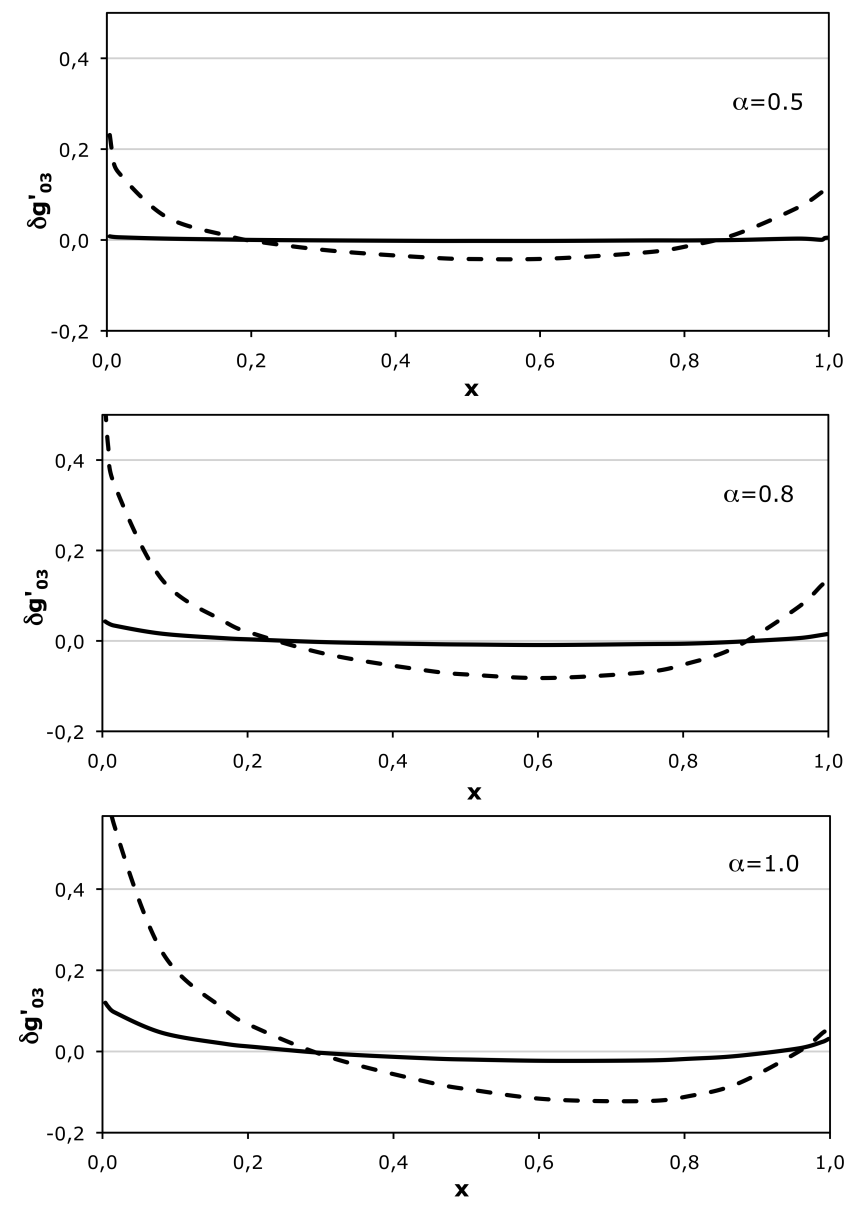

FIG. 13: $x$-dependence of the bare coupling constant $g_{03}^{\prime}$, calculated relatively to its mean value over the interval $x \in[0,1]$, for $\alpha=0.5$ (upper plot), $\alpha=0.8$ (middle plot) and $\alpha=1.0$ (lower plot), calculated for $\mu_{1}=100$. The solid (dashed) lines correspond to the results obtained with (without) the $f f \bar{f}$ Fock sector contribution.

The fact that $g_{03}^{\prime}(x)$ and $Z_{\omega}^{\prime}(x)$ are close to con-
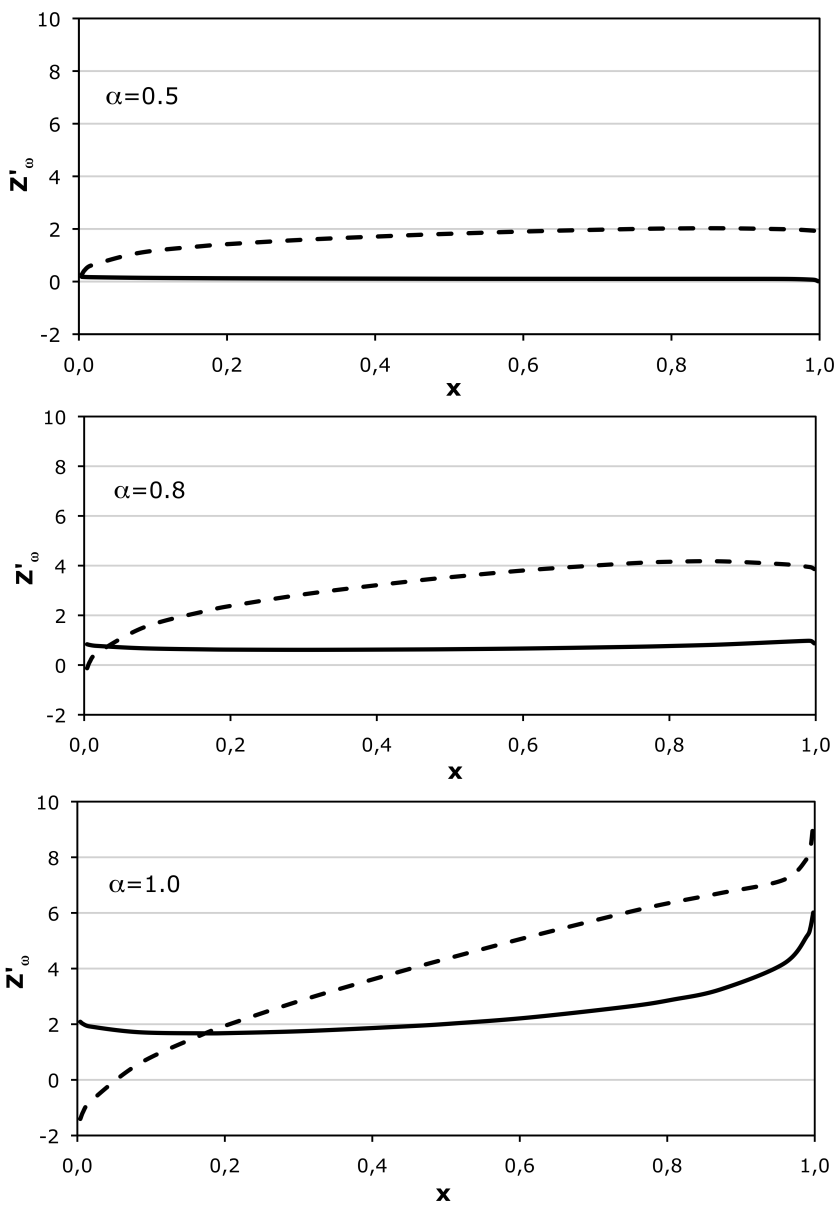

FIG. 14: $x$-dependence of the counterterm $Z_{\omega}^{\prime}$ for $\alpha=0.5$ (upper plot), $\alpha=0.8$ (middle plot), and $\alpha=1.0$ (lower plot), calculated for $\mu_{1}=100$. The solid (dashed) lines correspond to the results obtained with (without) the $f f \bar{f}$ Fock sector contribution.

stants is not a specific property of the Yukawa model, since we already encountered similar features in our studies of the scalar model [6. In contrast to the latter, where we incorporated particle-antiparticle loop contributions as well, we reproduced the property $g_{03}^{\prime}(x) \approx$ const in the Yukawa model within the quenched approximation. From here it follows that namely the contribution shown in Fig. 11(a) is responsible for this property. This was the main reason of using here the quenched approximation which allows us to keep such an important property of the bare parameters, on the one hand, and to retain the renormalization condition (16) and avoid complications connected 
with the fermion-antifermion loop renormalization, on the other hand.

For a deeper understanding of what happens with the bare parameters, when the antifermion d.o.f. are involved, let us consider the following simple approximation. It is instructive to solve the equation in Fig. 12 by iterations, taking, as the zero order approximation, the value of the two-body vertex function obtained for the $f+f b$ Fock space truncation, i. e. with $\Gamma_{2}=g$. We then substitute this value on the right-hand side of this equation and calculate the first iteration, $\Gamma_{2}\left(R_{\perp}, x\right)$, in the limit $m_{1} \rightarrow \infty$. We can then calculate $\Gamma_{2}\left(R_{\perp}^{*}(x), x\right)$ [see Eq. $[19)$ ], with both constituent particle legs corresponding to the physical particles $(i=j=0)$. This just determines the functions $b_{1,2}\left(R_{\perp}^{*}(x), x\right)$ which enter into the renormalization conditions (15) and (16). Since we are interested in the $x$-dependence of these functions, it is enough to calculate the contributions to the latter ones from the diagrams shown in Fig. 11. By direct calculation, we find that the sum of these two contributions to the function $b_{1}$ (denoted by $b_{1}^{a}$ and $b_{1}^{b}$ ) on the energy shell does not depend on $x$ :

$$
b_{1}^{a}\left(R_{\perp}^{*}(x), x\right)+b_{1}^{b}\left(R_{\perp}^{*}(x), x\right)=\text { const },
$$

while the total value of $b_{2}$ is zero:

$$
b_{2}^{a}\left(R_{\perp}^{*}(x), x\right)+b_{2}^{b}\left(R_{\perp}^{*}(x), x\right)=0 .
$$

So, on the level of the first iteration, we can meet the renormalization condition 16 exactly, with $g_{03}^{\prime}$ independent of $x$. The other condition (15) is satisfied automatically, without the need of an additional counterterm (20), i.e. $Z_{\omega}(x)=0$. Note that the counterterm $Z_{\omega}^{\prime}$ defined by Eq. 26b does not turn into zero, because it includes, beside $Z_{\omega}$, the contribution from the one-body state. We can assert only that in the approximation discussed above it becomes a constant.

This result has a simple explanation. The diagrams shown in Fig. 11 with a constant internal two-body vertex $\Gamma_{2}=g$ coincide with the light-front perturbative ones taken in the order $g^{3}$. Since there are no other $g^{3}$-order perturbative contributions to the twobody vertex function, the sum of their amplitudes, on the energy shell, is identical to the corresponding on-mass-shell Feynman amplitude which is a constant and does not depend on $\omega$. Eqs. 55 and 56 are direct consequences of this fact.

We may continue iterating the equation in Fig. 12 and represent each of the functions $b_{1,2}$ as a series in powers of the coupling constant. But these expansions, starting with the order $g^{5}$, differ from the perturbative ones. Indeed, perturbative contributions involve Fock sectors with arbitrary number of particles (of course, only those which are compatible with the order of perturbation), while the nonperturbative equation for $\Gamma_{2}$ contains no contributions from higher than three-body Fock sectors. As a result, $b_{1,2}\left(R_{\perp}^{*}(x), x\right)$ are no more constants, i. e. they depend on $x$. In order to enforce the fulfilment of the renormalization conditions, we have to introduce $x$ dependent bare parameters $g_{03}^{\prime}(x)$ and $Z_{\omega}^{\prime}(x)$. The same happens in our nonperturbative calculations, where the two-body vertex function $\Gamma_{2}$ is far from being a constant. Note that if we assigned different vertex factors to the elementary vertices $f \rightarrow f+b$ and $b \rightarrow f+\bar{f}$, we would not get the properties 55 and (56).

In the $f+f b+f b b$ approximation, the $x$-dependence of $b_{1,2}\left(R_{\perp}^{*}(x), x\right)$ is governed by the diagram in Fig. 11(b) only. The lowest order iterative contribution of the latter diagram to the function $b_{2}$, at $\mu_{1} \gg\{m, \mu\}$, has the form

$$
b_{2}^{b}\left(R_{\perp}^{*}(x), x\right)=-\frac{g^{3}}{4 \pi^{2}} \log \frac{\mu_{1}}{\mu}+f_{2}(x),
$$

where $f_{2}(x)$ is a function of $x$ and it does not depend on $\mu_{1}$. In order to save the renormalization condition (15), we have to add the structure (20) to the interaction Hamiltonian, with the $x$-dependent counterterm $Z_{\omega}(x)=-b_{2}^{b}\left(R_{\perp}^{*}(x), x\right)$ which has a constant $\mu_{1}$-dependent part (divergent when $\mu_{1} \rightarrow \infty$ ) and a finite $x$-dependent part. For higher order iterations, terms divergent when $\mu_{1} \rightarrow \infty$ appear in the $x$-dependent part of the counterterm as well. Hence, in our nonperturbative calculations within the $f+f b+f b b$ truncated space, we do expect strong $x$-dependence of the bare parameters, which is confirmed by the results represented in Figs. 13 and 14 .

We can now proceed to the calculation of the electromagnetic form factors. The general method is the same as in Sec. IVB, With the antifermion d.o.f. included in the quenched approximation, one should take into account the additional contributions to the EMV, shown in Fig. 15. The amount of computations can be reduced by exploiting some symmetry properties of the diagrams, since the two contributions in Fig. 15(a) are exactly the same. So, we can calculate only one of them and multiply the result by a factor of 2 . 

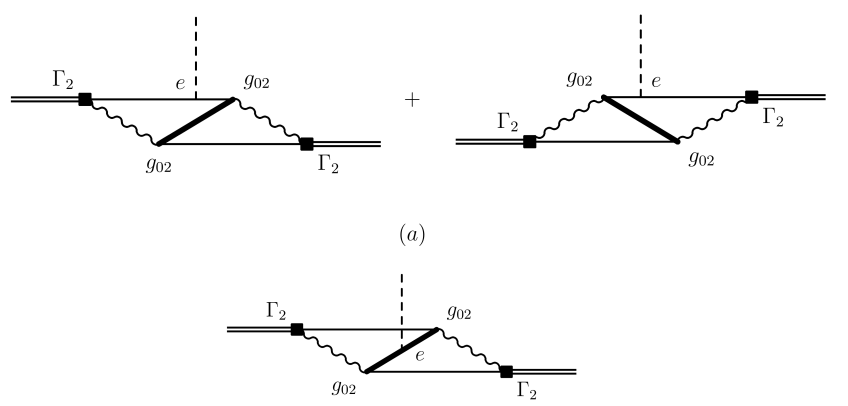

(b)

FIG. 15: Antifermion contributions to the electromagnetic vertex in the quenched approximation.

For numerical calculations, we take the same values of the particle masses and the coupling constant as in Sec. IV C, Our results for the fermion AMM are shown in Fig. 16. Although the antifermion d.o.f. have drastic influence on the $x$-dependence of the bare parameters, they decrease the AMM value, as compared to that in the $f+f b+f b b$ approximation (see Fig. 6), by only $\sim 2 \%$ for $\alpha=0.5, \sim 4 \%$ for $\alpha=0.8$, and $\sim 8 \%$ for $\alpha=1.0$. So small influence of the $f f \bar{f}$ Fock sector on the AMM is caused by the smallness of the ratio $\mu / m=0.147$. At larger $\mu$ the effect of the antifermion d.o.f. reveals itself stronger. The AMM is stable, as a function of $\mu_{1}$, if the latter is much larger than the physical masses, but not too close to the critical mass $\mu_{1 c}$ which is the same as in the $f+f b+f b b$ approximatoin (see Sec. IV C). If the latter condition is violated, we recover the AMM instability similar to the one obtained in the $f+f b+f b b$ truncation, with however a larger amplitude. A visible deviation of the AMM from the constant value is seen on the lower plot in Fig. 16, when $\mu_{1}>200$, while $\mu_{1 c}$ for $\alpha=1$ is about $1800\left[\log _{10}\left(\mu_{1 c}^{2} / \mu^{2}\right) \approx 8.2\right]$. For $\alpha=0.5$ and $\alpha=0.8\left[\log _{10}\left(\mu_{1 c}^{2} / \mu^{2}\right) \approx 19.1\right.$ and $\approx 11.0$, respectively] the AMM deviations from the constant in the interval $100<\mu_{1}<1000$ do not exceed the computational precision level $(\sim 0.5 \%)$. This means that with the same accuracy we have no any sign of uncanceled divergences.
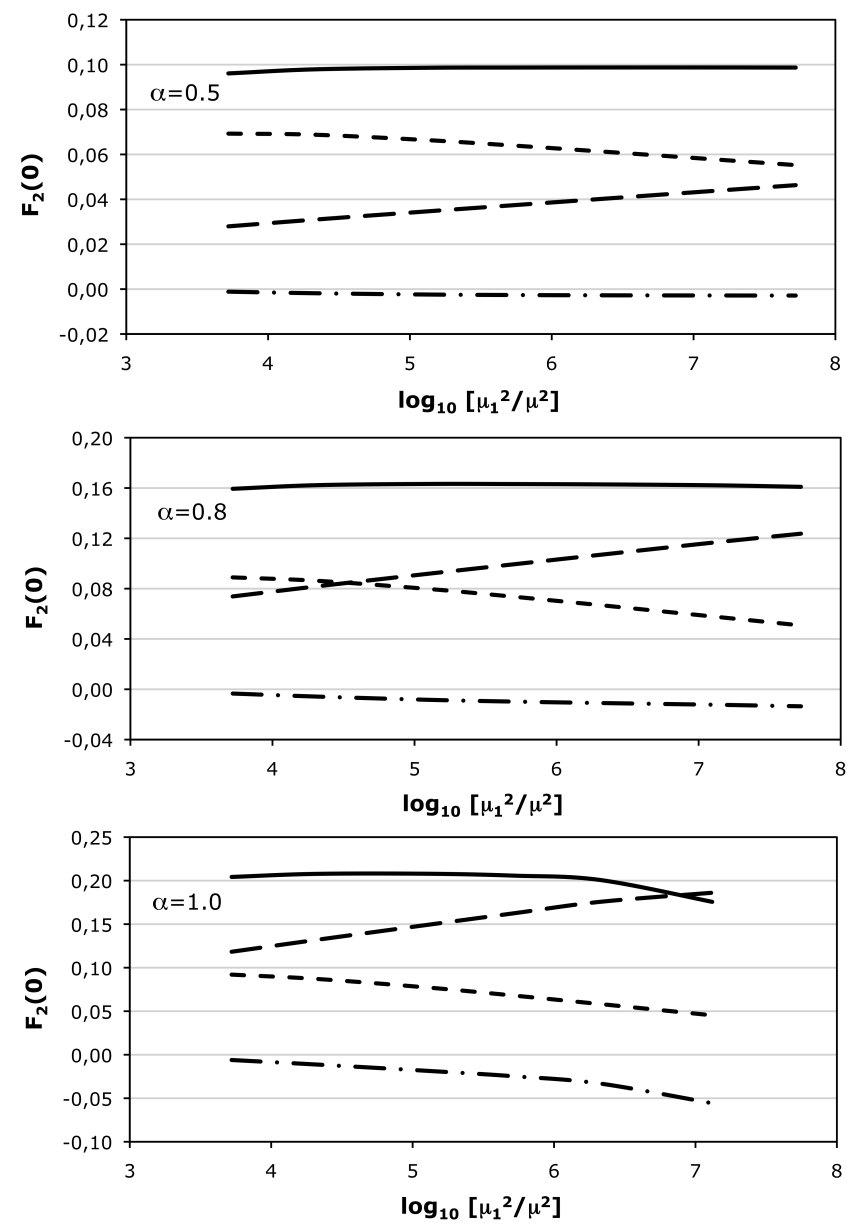

FIG. 16: The same as in Fig. 6, but including the contributions from antifermion d.o.f. (dash-dotted line), shown in Fig. 15

\section{CONCLUSION}

The results reported in this study are a first example of a full, nonperturbative, calculation of the properties of relativistic compound systems in the FSDR framework. The general approach is based on an expansion of the state vector of the system considered in Fock components, within LFD. The use of CLFD, together with an appropriate renormalization scheme in truncated Fock space, gives a very promising opportunity to calculate properties of compound systems in a regularization scale invariant way.

The full implementation of the renormalization conditions, which relate the on-energy-shell two-body vertex function to physical observables, is the last build- 
ing block in our strategy to get reliable predictions for physical observables in the nonperturbative domain. It leads a priori, and unambiguously, to the dependence of the bare parameters on one of the kinematical variables of the two-body vertex function, like for instance the longitudinal momentum fraction $x$. This dependence must disappear in an exact calculation, i.e. when the Fock space is not truncated.

We applied our strategy to the calculation of the fermion electromagnetic form factors in the Yukawa model, in the three-body Fock space truncation. Within our numerical precision the form factors, as a function of the momentum transfer, are independent of the regularization scale (the PV boson mass), as soon as the latter is large enough compared to the typical intrinsic energy/momentum scales of the system, but smaller than some critical value $\mu_{1 c}$.

We finally investigated the role of antifermion d.o.f. We showed that, in the leading order of perturbation theory, the contributions to the state vector of the Fock sector with an antifermion are precisely the ones which make the renormalization conditions fully consistent. In that case, no extra $\omega$-dependent counterterms are needed to restore the rotational invariance of the two-body vertex function on the energy shell, while the $x$-dependence of the bare parameters is canceled exactly. In our nonperturbative calculations, these contributions considerably improve the self-consistency of the renormalization conditions.

\section{Appendix A: The coefficients $\bar{c}_{0}, \bar{C}_{0}, \bar{c}_{0}^{\prime}$, and $\bar{C}_{0}^{\prime}$}

It is convenient to introduce the following notations:

$$
\begin{aligned}
\eta_{1}= & R_{\perp}^{\prime 2}+x^{2} m^{2}+\left(1-x^{\prime}\right) \mu_{j^{\prime}}^{2} \\
\bar{A}= & (1-x) x R_{\perp}^{\prime 2}+\left(1-x^{\prime}\right) x^{\prime} R_{\perp}^{2} \\
& +x x^{\prime}\left(2-x-x^{\prime}\right) m^{2} \\
\bar{B}= & 2(1-x)\left(1-x^{\prime}\right) R_{\perp}^{\prime} R_{\perp}, \\
\bar{D}= & 8 \pi^{2}
\end{aligned}
$$

and

$$
\begin{aligned}
& \bar{J}_{0}=\int_{0}^{2 \pi} \frac{d \phi^{\prime}}{2 \pi \bar{D}\left(\bar{A}+\bar{B} \cos \phi^{\prime}\right)}=\frac{\operatorname{sign}(\bar{A})}{\bar{D} \sqrt{\bar{A}^{2}-\bar{B}^{2}}}, \\
& \bar{J}_{1}=\int_{0}^{2 \pi} \frac{\cos \phi^{\prime} d \phi^{\prime}}{2 \pi \bar{D}\left(\bar{A}+\bar{B} \cos \phi^{\prime}\right)}=\frac{1}{\bar{D} \bar{B}}-\frac{\bar{A}}{\bar{B}} \bar{J}_{0} .
\end{aligned}
$$

Now the coefficients in Eqs. (54) take the form

$$
\begin{aligned}
\bar{c}_{0}= & \frac{(x-1) R_{\perp}^{\prime}}{R_{\perp} \eta_{1}}\left[R_{\perp} R_{\perp}^{\prime} \bar{J}_{0}\right. \\
& \left.+m^{2}\left(x x^{\prime}+2 x+2 x^{\prime}-4\right) \bar{J}_{1}\right], \\
\bar{C}_{0}= & \frac{(x-1) m^{2}}{R_{\perp} \eta_{1}}\left[R_{\perp}\left(3 x^{\prime}-2\right) \bar{J}_{0}-R_{\perp}^{\prime}(3 x-2) \bar{J}_{1}\right], \\
\bar{c}_{0}^{\prime}= & \frac{(x-1) R_{\perp}^{\prime}}{\eta_{1}}\left[R_{\perp}^{\prime}(3 x-2) \bar{J}_{0}-R_{\perp}\left(3 x^{\prime}-2\right) \bar{J}_{1}\right], \\
\bar{C}_{0}^{\prime}= & \frac{(x-1)}{\eta_{1}}\left[m^{2}\left(x x^{\prime}+2 x+2 x^{\prime}-4\right) \bar{J}_{0}\right. \\
& \left.+R_{\perp} R_{\perp}^{\prime} \bar{J}_{1}\right] .
\end{aligned}
$$

Note that these coefficients do not depend on the in$\operatorname{dex} j$.

\section{Acknowledgments}

Two of us (V. A. K. and A. V. S.) are sincerely grateful for the warm hospitality of the Laboratoire de Physique Corpusculaire, Université Blaise Pascal, in Clermont-Ferrand, where a part of the present study was performed, under a CNRS/RAS agreement.
[1] D. Bernard, Th. Cousin, V.A. Karmanov, and J.F. Mathiot, Phys. Rev. D 65, 025016 (2001).

[2] V.A. Karmanov, J.-F. Mathiot, and A.V. Smirnov. Phys. Rev. D 69, 045009 (2004).

[3] V.A. Karmanov, J.-F. Mathiot, and A.V. Smirnov, Phys. Rev. D 75, 045012 (2007).

[4] V.A. Karmanov, J.-F. Mathiot, and A.V. Smirnov,
Phys. Rev. D 77, 085028 (2008).

[5] V.A. Karmanov, J.-F. Mathiot, and A.V. Smirnov, Phys. Rev. D 82, 056010 (2010).

[6] J.-F. Mathiot, A.V. Smirnov, N.A. Tsirova, and V.A. Karmanov, Few-Body Syst. 49, 183 (2011).

[7] P.A.M. Dirac, Rev. Mod. Phys. 21, 392 (1949).

[8] S.J. Brodsky, H.C. Pauli, and S.S. Pinsky, Phys. Rep. 
301, 299 (1998)

[9] V.A. Karmanov, Zh. Eksp. Teor. Fiz. 71, 399 (1976) [Sov. Phys. JETP 44, 210 (1976)].

[10] J. Carbonell, B. Desplanques, V.A. Karmanov, and J.-F. Mathiot, Phys. Rep. 300, 215 (1998).

[11] S.J. Brodsky, J.R. Hiller, and G. McCartor, Phys. Rev. D 64, 114023 (2001);

S.J. Brodsky, J.R. Hiller, and G. McCartor, Ann. Phys. 305, 266 (2003);

S.J. Brodsky, J.R. Hiller and G. McCartor, Ann. Phys. 321, 1240 (2006).
[12] St. Glazek, A. Harindranath, S. Pinsky, J. Shigemitsu, and K. Wilson, Phys. Rev. D 47, 1599 (1993).

[13] R.J. Perry, A. Harindranath, and K.G. Wilson, Phys. Rev. Lett. 65, 2959 (1990);

R.J. Perry and A. Harindranath, Phys. Rev. D 43, 4051 (1991).

[14] J.R. Hiller and S.J. Brodsky, Phys. Rev. D 59, 016006 (1998).

[15] V.A. Karmanov and J.-F. Mathiot, Nucl. Phys. A602, 388 (1996). 